\title{
Impacto de la inversión extranjera directa en el PIB mexicano por país de origen y entidad federativa de destino
}

\author{
Isela Elizabeth Téllez-León* \\ Francisco Venegas-Martínez ${ }^{* *}$
}

(Recibido: agosto 2017/Aceptado: enero 2018)

\section{Resumen}

Esta investigación estudia dos aspectos fundamentales de la inversión extranjera directa (IED), el país de origen y el Estado de destino, y evalúa el impacto de ambas variables sobre el producto interno bruto (PIB) para el caso mexicano. Para ello, la descomposición de varianza de la tasa de crecimiento, proveniente de un modelo VAR, del PIB real se utiliza para estimar cuánto de la variabilidad en la tasa de crecimiento es explicada por la IED de cada Estado tomando en cuenta el país de origen. El principal hallazgo es que algunos Estados reciben montos considerables de IED, pero no explican una variabilidad grande del crecimiento económico; esto se debe, posiblemente, a eslabonamientos en la industria.

Palabras clave: inversión extranjera directa, crecimiento económico, modelos econométricos, México.

Clasificación JEL: F21, F43, C5.

\footnotetext{
* Profesora de la Escuela Superior de Economía, Instituto Politécnico Nacional. Correo electrónico: tellezelizabeth_2015@yahoo.com.

** Profesor-investigador de la Escuela Superior de Economía del Instituto Politécnico Nacional. Correo electrónico: fvenegas1111@yahoo.com.mx.
} 


\title{
Impact of direct foreign investment on the mexican GDP by country of origin and state of destination
}

\begin{abstract}
This paper is aimed at studying two fundamental aspects of Foreign Direct Investment (FDI), the country of origin and the State of destination, and assessing the impact of both variables on the Gross Domestic Product (GDP) for the Mexican case. To do this, the variance decomposition of the real GDP, coming from a VAR model, is used to estimate how much of the variability of the growth rate is explained by the IED of each State taking into account the country of origin. The main finding is that some States receive considerable amounts of FDI, but they do not explain a large variability in economic growth; this is, possibly, due to linkages in the industry.
\end{abstract}

Keywords: foreign direct investment, economic growth, econometric modeling, Mexico.

JEL classification: F21, F43, C5.

\section{Introducción}

La relación entre inversión extranjera directa (IED) y crecimiento del producto interno bruto (PIB) es un tema actual y relevante tanto para economías emergentes como para industrializadas. La IED es uno de los elementos fundamentales que coadyuvan a reducir la brecha entre economías subdesarrolladas y desarrolladas. En particular para economías como México, una parte considerable del diseño de la política económica debe orientarse hacia la atracción de mayores flujos de IED que promuevan los eslabonamientos de producción entre industrias con los que actualmente cuentan las economías industrializadas.

La literatura especializada sobre el nexo entre la IED y el PIB es, sin duda, abundante. Por ejemplo, Shaikh (2010) estima un modelo de datos panel para 47 países en desarrollo durante 1981-1999 y encuentra relaciones de causalidad en el sentido de Granger entre dichas variables. Asimismo, Choe (2003) analiza 19 países, desarrollados y en desarrollo, para indagar si la IED causa en el sentido de Granger el crecimiento del producto durante el periodo 1971-1995. También, Nair-Reichert y Weinhold (2001) estudian, mediante un 
modelo de datos panel, 24 países en desarrollo durante 1971-1995, y encuentran que en promedio la IED tiene un impacto estadísticamente significativo sobre el crecimiento económico. Aunque los modelos panel que se han estimado en las investigaciones anteriores aportan resultados que son representativos del promedio y mejoran el entendimiento sobre la relación entre la IED y el crecimiento económico, éstos no consideran los efectos de la heterogeneidad entre países. Por esta razón, en la literatura también se encuentran modelos alternativos que enmiendan parcialmente dicha limitación a través de la metodología de vectores autorregresivos (VAR). Al respecto, Ericsson y Manuchehr (2001) estiman un modelo VAR para el caso de Dinamarca, Finlandia, Noruega y Suecia con el fin de estudiar la causalidad entre la IED y el crecimiento económico con base en el procedimiento propuesto por Toda y Yamamoto (1995) y Yamada y Toda (1998). No obstante, pocos son los trabajos que profundizan sobre el impacto de la IED en el crecimiento por regiones y/o entidades federativas dentro un país. La presente investigación estima la descomposición de varianza de la tasa de crecimiento del PIB real proveniente de un modelo VAR. Esta descomposición se utiliza para estimar cuánto de la variabilidad en la tasa de crecimiento es explicada por la IED de cada Estado tomando en cuenta tanto el país de origen. Particularmente, se estima la descomposición de varianza de la tasa de crecimiento del PIB real por región o estado de la república mexicana dimensionando por población y superficie de cada región o estado.

Es importante mencionar que a partir de 2014 hay estados con actualizaciones pendientes, lo que limita el horizonte de estudio. Los resultados obtenidos son un aporte en el estudio de los flujos de inversión en México porque no se encontró en la literatura un estudio similar para el caso de México. Se pueden encontrar otras investigaciones con enfoques distintos y objetivos como: Romero (2012) que realiza un análisis de cointegración para estudiar el impacto de la IED sobre la productividad del trabajo; Nunnenkamp y Alatorre (2007) que investigan sobre los efectos de la IED en el empleo; Waldkirch (2008) que examina el efecto de la IED en el crecimiento en México a partir de la firma del Tratado de Libre Comercio de Norte América; y Márquez-Pozos, Islas-Camargo y Venegas-Martínez (2003) que estudian la dinámica de los flujos internacionales de capital durante 1989-1999.

La presente investigación se distingue de la literatura existente en lo siguiente: 1) mejora sustancialmente el entendimiento sobre el impacto de la IED por estado o región en el crecimiento económico de México; 2) realiza un análisis de las compañías involucradas en la IED por origen y destino, 
lo cual permitirá dimensionar el monto de IED y número de compañías con IED por Estado de la República para crear o mejorar los eslabonamientos que se generan entre industrias; $y, 3$ ) proporciona recomendaciones que permiten a las autoridades derivar implicaciones y recomendaciones para el diseño de política económica que hagan más eficiente la captación, adquisición y spillovers de la IED en los diferentes estados de la república.

Esta investigación se encuentra organizada en las siguientes secciones: en la próxima sección de presenta un análisis por países de origen de IED a México; la tercera sección presenta la estimación de la descomposición de varianza; la cuarta sección realiza un análisis del número de sociedades de inversión extranjera; por último, se presentan las conclusiones y diversas recomendaciones de política económica.

\section{Países de origen de la IED en México, 1999-2013}

México ha sido uno de los principales receptores de IED debido a su proximidad con EU y al Tratado de Libre Comercio de Norte América (TLCAN). ${ }^{1}$ Es un hecho estilizado que las relaciones comerciales implican beneficios para ambas economías (Yeaple, 2005). La IED proveniente de EU hacia México siempre ha sido considerable, tan sólo de 1999 a 2013, ésta asciende a 168176.7 millones de dólares. La gráfica 1 presenta dos diagramas de pastel, el diagrama A muestra a los principales países que envían IED a México y la gráfica B muestra los principales países de América Latina (AL) que envían IED a México por orden de monto. Con base en lo anterior se observa que EU, Países Bajos, España, Canadá y Bélgica invierten montos importantes en México. En tanto que en América Latina (AL), Brasil, Panamá, Argentina, Colombia y Chile invierten un monto considerable en México.

\footnotetext{
${ }^{1}$ Para dimensionar la relación entre México y EU véase Blomstrom y Kokko (1997).
} 


\section{Gráfica 1 \\ IED por país de origen}

(A)

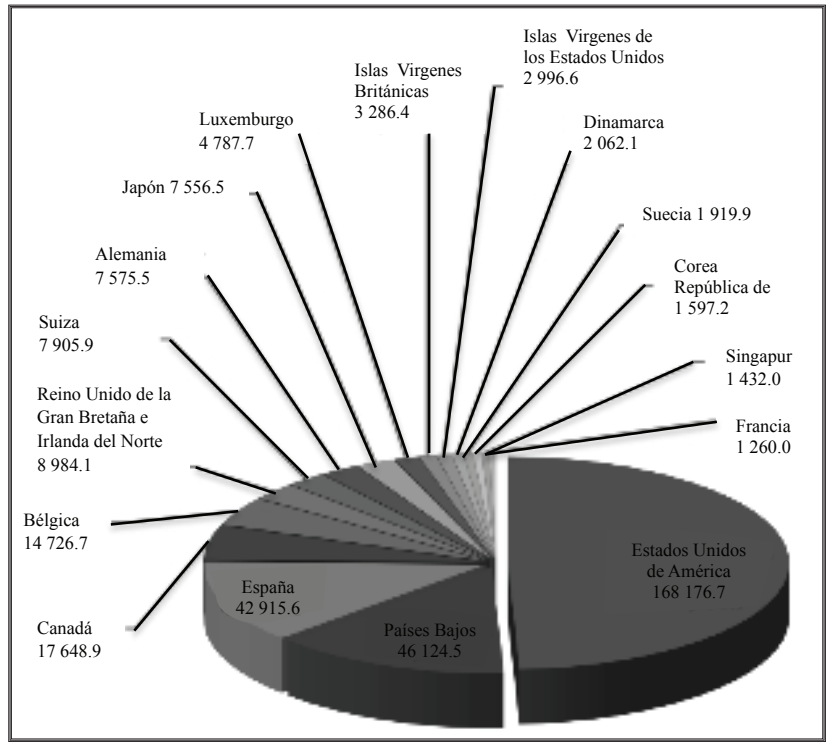

(B)

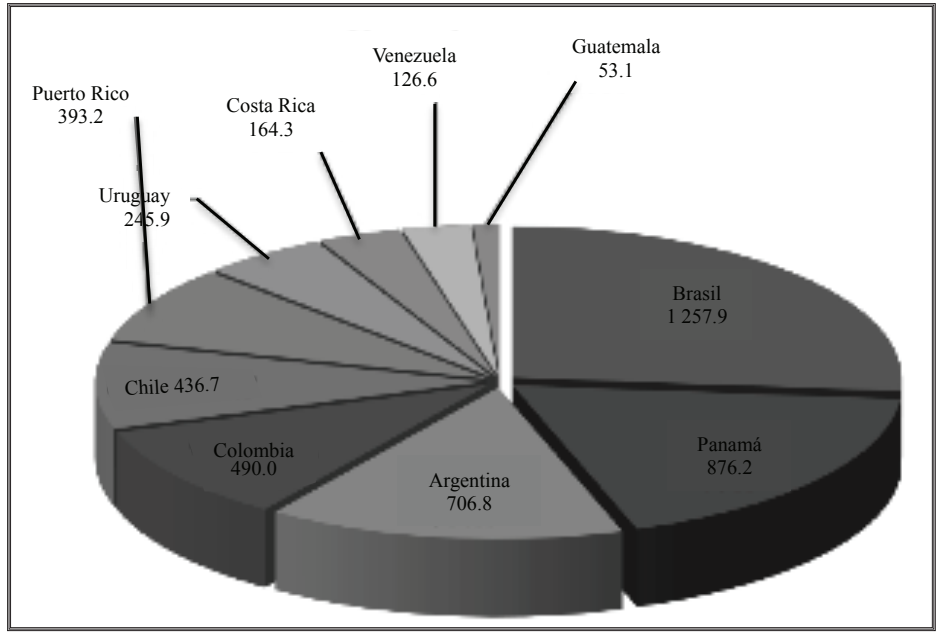

Fuente: elaboración propia con datos de la Secretaría de Economía. 
Con base en el informe sobre Inversión de la UNCTAD (2013) se observa que por primera, en 2012, los países en desarrollo absorbieron más IED que los países desarrollados con un total de $52 \%$ de los flujos mundiales de IED; este movimiento fue analizado por Basave y Gutiérrez (2013). Estos autores encuentran que el desplazamiento de los flujos de inversión se da después de la crisis económica de 2008. Esto en virtud de que los flujos de IED hacia países en desarrollo generan una mayor eficiencia en la producción por la combinación de capital humano y la tecnología transferida.

El informe de la UNCTAD (2013) señala que las economías en desarrollo generan casi un tercio de las salidas mundiales de IED con una tendencia positiva constante. Para tener una visión global de los flujos de IED se presentan en el apéndice A varios cuadros elaborados con información de la UNCTAD, en 1970-2012, sobre la estructura porcentual de la entrada de IED por bloques económicos, por características similares y por tratados comerciales. Dicho apéndice presenta, primero, la estructura porcentual de la entrada de IED en el mundo por bloques económicos. En dicho cuadro se observa que del total de los flujos mundiales alrededor del $41.5 \%$ se dirige a economías desarrolladas en 2012.

Este porcentaje ha ido disminuyendo desde 2000. Posteriormente, el apéndice A muestra la estructura porcentual de la entrada de IED por bloques de países que tienen características similares. El cuadro correspondiente, en el apéndice $\mathrm{A}$, contiene hechos relevantes, por ejemplo, los países con mayor endeudamiento no presentan más de 3\% de entrada de IED desde 1970, lo que implica que las economías sólidas y con fundamentos macroeconómicos sanos están atrayendo más capitales. Otro hecho importante es que economías con altos ingresos atraen IED, por ejemplo en 2012 dichas economías atrajeron el $31 \%$ de la IED.

Las economías emergentes desde 1980 atraen más del 10\% de la IED mundial disminuyendo en algunos años críticos. Es importante notar que los principales países exportadores de productos manufactureros atraen más del 50\% de los flujos de IED mundiales. En algunos años el porcentaje es muy alto (cerca de 90\%). Por último, en el apéndice A se destaca que los tratados comerciales tienen una estrecha relación con la IED. Entre las regiones que pertenecen a los siguientes tratados se observa el mayor porcentaje de atracción de la IED mundial: APEC (Asia-Pacific Economic Cooperation), EU27 (European Union), Euro Area, FTAA (Free Trade Area of the Americas), G8, G20, G77, GSTP (Global System of Trade Preferences), IDB (Islamic 
Development Bank), NAFTA (North American Free Trade Agreement), OAS (Organization of American States), entre otros. En la siguiente sección se examina la IED por entidad federativa con la finalidad de evaluar el impacto de la IED sobre el crecimiento económico en los diferentes estados del país.

\section{Análisis econométrico por región y entidad federativa}

En esta sección se analiza la IED por estados de la república mexicana, los cuales fueron agrupados, primero, por regiones para obtener una mejor visión del destino que tiene la IED a México. Esto permitirá observar cuáles son las regiones a las que se dirige la IED. Además se realiza un análisis econométrico a través de la descomposición de varianza del crecimiento económico por estado para conocer cuál de las entidades federativas explica una mayor variabilidad de la tasa de crecimiento del PIB. El análisis inicia con la región noreste de México (Coahuila, Nuevo León y Tamaulipas) la cual tiene a la segunda entidad federativa que recibió más IED dentro del periodo 1999-2013, Nuevo León con 33 mil 202.3 millones de dólares, véase el cuadro 1.

Cuadro 1

Noreste de México

\begin{tabular}{|cccc|}
\hline Entidad federativa & $\begin{array}{c}\text { IED, 1999-2013 } \\
\text { (millones de } \\
\text { dólares) }\end{array}$ & Población, 2010/1 & Superficie $\left(\mathbf{k m}^{\mathbf{2})} \mathbf{P}^{\mathbf{2}}\right.$ \\
\hline Coahuila & 4899.5 & 2748.391 & 151.445 \\
Nuevo León & 33202.3 & 4653.458 & 64.203 \\
Tamaulipas & 6085.6 & 3268.554 & 80.148 \\
\hline
\end{tabular}

${ }^{*}$ Cifras sujetas a cambios, por las continuas revisiones. ${ }^{1 /}$ INEGI: Censo de Población y Vivienda 2010. ²/ INEGI. Marco Geo-estadístico 2005.

Fuente: elaboración propia con datos publicados por la Secretaría de Economía e INEGI.

El estado dentro de la región en estudio que explica una mayor variabilidad en la tasas de crecimiento del PIB real es Coahuila, véase el cuadro 2, lo cual sorprende debido a que no ha recibido un monto de IED tan importante como el de Nuevo León. Sin embargo, al revisar con mayor detalle se 
observa que el sector económico de destino fue, principalmente, la industria manufacturera (la fabricación de maquinaria, equipo y fabricación de equipo de transporte), la minería y la industria de las bebidas y del tabaco. Lo anterior apunta a que el destino por sector económico explica, con detalle, la variabilidad en el crecimiento económico, ya que por sector económico se observa que los eslabonamientos y la contribución de la IED a la productividad están relacionados con las condiciones preexistentes en el sector ya que esto depende de la capacidad de absorción de la tecnología.

\section{Cuadro 2}

Descomposición de la varianza del $\triangle$ PIBreal $^{*}$ noreste de México

\begin{tabular}{|ccccc|}
\hline Periodo & PIB_real & IED_Coahuila & IED_NL & IED_Tamp. \\
\hline 1 & 100.0000 & 0.000000 & 0.000000 & 0.000000 \\
2 & 96.32676 & 3.630445 & 0.031291 & 0.011503 \\
3 & 96.43139 & 3.451860 & 0.090623 & 0.026130 \\
4 & 96.31315 & 3.542644 & 0.118361 & 0.025848 \\
5 & 96.30265 & 3.539308 & 0.128622 & 0.029424 \\
6 & 96.28708 & 3.546184 & 0.129555 & 0.037181 \\
7 & 96.28409 & 3.544924 & 0.129493 & 0.041497 \\
8 & 96.27973 & 3.547242 & 0.129644 & 0.043379 \\
\hline
\end{tabular}

Cholesky Ordering: PIBreal, IED_Coahuila, IED_NL, IED_Tamaulipas. Errores estándar: Monte Carlo (1,000,000 repetitions). *Cifras sujetas a cambios, por las continuas revisiones. Fuente: elaborada con datos publicados por la Secretaría de Economía.

La región noroeste (comprendida por Baja California, Baja California Sur, Chihuahua, Durango, Sinaloa y Sonora) presenta dos Estados con montos considerables de IED, durante 1999-2013, estos son Chihuahua (17 mil 467.9 millones de dólares) y Baja California (13 mil 842.5 millones de dólares), cuadro 3. 


\section{Cuadro 3 \\ Noroeste de México}

\begin{tabular}{|lccc|}
\hline Entidad federativa & $\begin{array}{c}\text { IED, 1999-2013 } \\
\text { (millones de } \\
\text { dólares) }\end{array}$ & Población (2010)/ ${ }^{\mathbf{2}}$ & Superficie $\left(\mathbf{k m}^{\mathbf{2}}\right)^{\mathbf{2}}$ \\
\hline \hline Baja California & 13842.5 & 3155.070 & 71.546 \\
Baja California Sur & 4878.3 & 637.026 & 73.943 \\
Chihuahua & 17467.9 & 3406.465 & 247.487 \\
Durango & 2550.0 & 1632.934 & 123.367 \\
Sinaloa & 1147.7 & 2767.761 & 57.331 \\
Sonora & 4499.3 & 2662.480 & 179.516 \\
\hline
\end{tabular}

*Cifras sujetas a cambios, por las continuas revisiones. ${ }^{1 /}$ INEGI: Censo de Población y Vivienda 2010. ${ }^{2} /$ INEGI. Marco Geo-estadístico 2005.

Fuente: elaboración propia con datos publicados por la Secretaría de Economía e INEGI.

Durango y Sonora explican en mayor medida la variabilidad en la tasa de crecimiento del PIB real, cuadro 4. Durango tiene mayor IED dirigida a minería, servicios financieros, seguros y comercio. Mientras que, Sonora presenta mayor IED en minería, industrias manufacturera y servicios de alojamiento temporal, de preparación de alimentos y bebidas.

\section{Cuadro 4}

Descomposición de la varianza del $\Delta$ PIBreal ${ }^{*}$ Noroeste de México

\begin{tabular}{|ccccccc|}
\hline \multirow{2}{*}{ Periodo } & PIB_real & $\begin{array}{c}\text { IED_Baja } \\
\text { California }\end{array}$ & $\begin{array}{c}\text { IED_- } \\
\text { Chihuahua) }\end{array}$ & $\begin{array}{c}\text { IED_ } \\
\text { Durango }\end{array}$ & $\begin{array}{c}\text { IED_- } \\
\text { Sinaloa }\end{array}$ & $\begin{array}{c}\text { IED_- } \\
\text { Sonora }\end{array}$ \\
\hline \hline 1 & 100.0000 & 0.000000 & 0.000000 & 0.000000 & 0.000000 & 0.000000 \\
2 & 41.25458 & 11.57184 & 2.362725 & 26.78979 & 1.297323 & 16.72373 \\
3 & 28.36376 & 11.51786 & 3.910160 & 30.22176 & 2.493622 & 23.49283 \\
4 & 23.21275 & 10.79794 & 4.193940 & 32.34027 & 3.217836 & 26.23726 \\
5 & 20.95660 & 10.07737 & 4.457486 & 33.31834 & 3.624973 & 27.56522 \\
6 & 19.91882 & 9.519571 & 4.649342 & 33.84986 & 3.854295 & 28.20812 \\
7 & 19.36969 & 9.100559 & 4.805928 & 34.16134 & 3.996014 & 28.56647 \\
8 & 19.05876 & 8.785029 & 4.930292 & 34.35822 & 4.088795 & 28.77891 \\
\hline
\end{tabular}

Cholesky Ordering: PIB_real, IED_Baja California, IED_Chihuahua, IED_Durango, IED_Sinaloa, IED_Sonora. Errores estándar: Monte Carlo (1,000,000 Repetitions). "Cifras sujetas a cambios, por las continuas revisiones.

Fuente: elaboración propia con datos publicados por la Secretaría de Economía. 
La región de occidente (representada por Colima, Nayarit, Michoacán y Jalisco) muestra a Jalisco como la entidad con mayor IED recibida, dentro del periodo 1999-2013, mientras que las otras tres entidades registran niveles de IED relativamente pequeñas, obsérvese el cuadro 5.

\section{Cuadro 5}

Occidente de México

\begin{tabular}{|lccc|}
\hline $\begin{array}{c}\text { Entidad } \\
\text { federativa }\end{array}$ & $\begin{array}{c}\text { IED, 1999-2013 } \\
\text { (millones de dólares) }\end{array}$ & Población (2010)/1 & Superficie $\left(\mathbf{k m}^{\mathbf{2}}\right) \mathbf{/}^{\mathbf{2}}$ \\
\hline Colima & 254.5 & 650.555 & 5.627 \\
Nayarit & 1265.5 & 1084979 & 27.862 \\
Michoacán & 1856.8 & 4351.037 & 58.667 \\
Jalisco & 11485.4 & 7350.682 & 78.630 \\
\hline
\end{tabular}

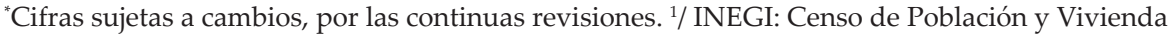
2010. 2/ INEGI. Marco Geo-estadístico 2005.

Fuente: elaboración propia con datos publicados por la Secretaría de Economía e INEGI

La variabilidad de la tasa de crecimiento del PIB real se explica principalmente por Nayarit, véase el cuadro 6. Los sectores económicos a los que se dirige la IED en Nayarit son: servicios de alojamiento temporal, de preparación de alimentos y bebidas, servicios inmobiliarios y de alquiler de bienes muebles e intangibles, comercio, servicios financieros y de seguros.

\section{Cuadro 6}

Descomposición de la varianza del $\triangle$ PIBreal $^{*}$ occidente de México

\begin{tabular}{|cccccc|}
\hline Periodo & PIB_real & IED_Colima & ED_Nayarit & IED_Michoacán & IED_Jalisco \\
\hline \hline 1 & 100.0000 & 0.000000 & 0.000000 & 0.000000 & 0.000000 \\
2 & 61.89226 & 0.190261 & 34.95848 & 2.040597 & 0.918405 \\
3 & 51.14717 & 0.807120 & 29.21210 & 18.14037 & 0.693236 \\
4 & 47.57248 & 3.980521 & 30.50175 & 15.12331 & 2.821944 \\
5 & 49.19219 & 3.661410 & 28.09357 & 15.50985 & 3.542973 \\
6 & 45.07384 & 4.180650 & 31.25755 & 15.73985 & 3.748106 \\
7 & 45.92900 & 4.020552 & 29.73976 & 16.83240 & 3.478282 \\
8 & 45.17485 & 3.892483 & 31.56373 & 16.04711 & 3.321825 \\
\hline
\end{tabular}

Cholesky Ordering: PIB_real, IED_Colima, IED_Nayarit, IED_Michoacán y IED_Jalisco. Errores estándar: Monte Carlo (1 000000 Repetitions). 'Cifras sujetas a cambios, por las continuas revisiones. Fuente: elaboración propia con datos publicados por la Secretaría de Economía. 
Puebla encabeza a los estados con mayor recepción de IED, durante 19992013, dentro de la región oriente, cuadro 7. Precisamente, Puebla explica una mayor proporción de la variabilidad del crecimiento económico, cuadro 8.

\section{Cuadro 7}

oriente de México

\begin{tabular}{|lccc|}
\hline Entidad federativa & $\begin{array}{c}\text { IED, 1999-2013 } \\
\text { (millones de } \\
\text { dólares) }\end{array}$ & Población (2010)/ ${ }^{*}$ & Superficie $\left(\mathbf{k m}^{2}\right) \mathbf{P}^{\mathbf{2}}$ \\
\hline Hidalgo & 79.1 & 2665.018 & 20.856 \\
Puebla & 6819.5 & 5779.829 & 34.251 \\
Tlaxcala & 539.6 & 1169.936 & 3.997 \\
Veracruz & 1205.4 & 7643.194 & 71.856 \\
\hline
\end{tabular}

"Cifras sujetas a cambios, por las continuas revisiones. ${ }^{1 /}$ INEGI: Censo de Población y Vivienda 2010. ²/ INEGI. Marco Geo-estadístico 2005.

Fuente: elaboración propia con datos publicados por la Secretaría de Economía e INEGI.

Asimismo, Puebla es uno de los Estados que han recibido históricamente montos considerables de IED y que explican la mayor variabilidad de la tasa de crecimiento con respecto de otros Estados de la Región en análisis.

\section{Cuadro 8}

Descomposición de la varianza del $\triangle$ PIBreal $^{*}$ oriente de México

\begin{tabular}{|cccccc|}
\hline Periodo & PIB_real & IED_Hidalgo & IED_Puebla & IED_Tlaxcala & IED_Veracruz \\
\hline \hline 1 & 100.0000 & 0.000000 & 0.000000 & 0.000000 & 0.000000 \\
2 & 39.20653 & 2.433244 & 36.40457 & 1.873707 & 21.95566 \\
3 & 18.70995 & 1.310780 & 68.23422 & 1.821750 & 11.74505 \\
4 & 14.82416 & 1.387987 & 69.65832 & 1.805455 & 14.12953 \\
5 & 14.13895 & 1.293575 & 71.91431 & 1.834799 & 12.65316 \\
6 & 11.99233 & 1.463363 & 73.29873 & 1.840402 & 13.24558 \\
7 & 12.41473 & 1.441885 & 73.12898 & 1.846375 & 13.01440 \\
8 & 11.01079 & 1.375365 & 74.81978 & 1.847271 & 12.79407 \\
\hline \hline
\end{tabular}

Cholesky Ordering: PIB_real, IED_Hidalgo, IED_Puebla, IED_Tlaxcala, IED_Veracruz. Standard Errors: Monte Carlo (1 000000 repetitions). ${ }^{*}$ Cifras sujetas a cambios, por las continuas revisiones. Fuente: elaboración propia con datos publicados por la Secretaría de Economía. 
En el periodo de estudio, la región centro-norte de México (integrada por: Aguascalientes, Guanajuato, Querétaro, San Luis Potosí y Zacatecas) presentó montos de IED de magnitud similar. En este sentido, el porcentaje de a variabilidad en la tasa de crecimiento del PIB que explican las Entidades referidas, de la región centro-norte, son aproximadamente, 4\%, 7\%, $2 \% 5 \%$ y $3 \%$, respectivamente; véanse los cuadros 9 y 10 , en ese orden.

\section{Cuadro 9}

Centro-norte de México

\begin{tabular}{|c|c|c|c|}
\hline Entidad federativa & $\begin{array}{c}\text { IED, 1999-2013 } \\
\text { (millones de } \\
\text { dólares) }\end{array}$ & Población (2010)/1 & Superficie $\left(\mathrm{km}^{2}\right) /^{2}$ \\
\hline Hidalgo & 79.1 & 2665.018 & 20.856 \\
\hline Puebla & 6819.5 & 5779.829 & 34.251 \\
\hline Tlaxcala & 539.6 & 1169.936 & 3.997 \\
\hline Veracruz & 1205.4 & 7643.194 & 71.856 \\
\hline
\end{tabular}

*Cifras sujetas a cambios, por las continuas revisiones. ${ }^{1 /}$ INEGI: Censo de Población y Vivienda 2010. ² INEGI. Marco Geo-estadístico 2005.

Fuente: elaborada con datos publicados por la Secretaría de Economía e INEGI.

\section{Cuadro 10}

Descomposición de la varianza del $\triangle$ PIBreal $^{*}$ centro-norte de México

\begin{tabular}{|ccccccc|}
\hline Periodo & PIB_real & $\begin{array}{c}\text { IED_ } \\
\text { Aguas } \\
\text { Calientes }\end{array}$ & $\begin{array}{c}\text { D IED_ } \\
\text { Guanajuato }\end{array}$ & $\begin{array}{c}\text { IED_ } \\
\text { Queretaro }\end{array}$ & $\begin{array}{c}\text { IED_- } \\
\text { San Luis } \\
\text { Posí }\end{array}$ & $\begin{array}{c}\text { IED_- } \\
\text { Zacatecas }\end{array}$ \\
\hline \hline 1 & 100.0000 & 0.000000 & 0.000000 & 0.000000 & 0.000000 & 0.000000 \\
2 & 85.64430 & 0.833047 & 2.733745 & 1.780943 & 6.156926 & 2.851036 \\
3 & 79.92877 & 3.816054 & 7.420913 & 1.570066 & 4.911498 & 2.352696 \\
4 & 79.26638 & 3.933908 & 7.374699 & 1.572665 & 5.073526 & 2.778827 \\
5 & 79.46802 & 3.980432 & 7.208689 & 1.532826 & 5.102667 & 2.707364 \\
6 & 79.23116 & 3.946511 & 7.403838 & 1.584171 & 5.073353 & 2.760964 \\
7 & 79.23156 & 3.963928 & 7.421300 & 1.576689 & 5.054722 & 2.751804 \\
8 & 79.22026 & 3.959722 & 7.418245 & 1.578652 & 5.065008 & 2.758118 \\
\hline
\end{tabular}

Cholesky Ordering: PIB_real, IED_Aguascalientes, IED_Guanajuato, IED_Querétaro, IED_San Luis Potosí, IED_Zacatecas. Errores estándar: Monte Carlo (1000000 repetitions). *Cifras sujetas a cambios, por las continuas revisiones.

Fuente: elaborada con datos publicados por la Secretaría de Economía. 
En la región centro-sur, la Ciudad de México encabeza la entidad con mayor recepción de IED durante 1999-2013, véase cuadro 11. La referida región también contiene al Estado de México que representa el tercer lugar, de los estados de la república mexicana, con mayor IED en el periodo 1999-2013.

\author{
Cuadro 11 \\ centro-sur de México
}

\begin{tabular}{|c|c|c|c|}
\hline Entidad federativa & $\begin{array}{c}\text { IED, 1999-2013* } \\
\text { (millones de dólares) }\end{array}$ & Población (2010)/ ${ }^{1}$ & Superficie $\left(\mathrm{km}^{2}\right){ }^{2}$ \\
\hline Ciudad de México & 194816.8 & 8851.080 & 1.484 \\
\hline Estado de México & 18831.8 & 15175.862 & 22.333 \\
\hline Morelos & 1565.8 & 1777.227 & 4.892 \\
\hline
\end{tabular}

${ }^{*}$ Cifras sujetas a cambios, por las continuas revisiones. ${ }^{1 /}$ INEGI: Censo de Población y Vivienda 2010. 2/ INEGI. Marco Geo-estadístico 2005.

Fuente: elaborada con datos publicados por la Secretaría de Economía e INEGI.

El cuadro 12 muestra que la variabilidad de la tasa de crecimiento del PIB real es explicada por los Estados que integran la región centro-sur, aunque no es tan grande como se esperaba. Los sectores hacia donde se dirige la IED en la Ciudad de México son: los servicios financieros y de seguros, el sector manufacturero, el comercio, el sector de servicios inmobiliarios y de alquiler de bienes muebles e intangibles, y el sector de la información en medios masivos, entre otros. 
Cuadro 12

Descomposición de la varianza del $\Delta$ PIBreal $^{*}$

centro-sur de México

\begin{tabular}{|ccccc|}
\hline Periodo & PIB_real & $\begin{array}{c}\text { IED_Ciudad } \\
\text { de México }\end{array}$ & $\begin{array}{c}\text { IED_Edo. } \\
\text { de México }\end{array}$ & IED_Morelos \\
\hline \hline 1 & 100.0000 & 0.000000 & 0.000000 & 0.000000 \\
2 & 94.04289 & 0.003611 & 5.320446 & 0.633054 \\
3 & 93.29869 & 0.455982 & 5.316630 & 0.928699 \\
4 & 93.01460 & 0.727385 & 5.237870 & 1.020140 \\
5 & 92.73263 & 1.000281 & 5.241786 & 1.025304 \\
6 & 92.62837 & 1.116489 & 5.230741 & 1.024402 \\
7 & 92.56841 & 1.167342 & 5.227288 & 1.036959 \\
8 & 92.54026 & 1.179918 & 5.227685 & 1.052137 \\
\hline
\end{tabular}

Cholesky Ordering: PIB_real, IED_Ciudad de México, IED_Edo. De Mex., IED_Morelos. Errores estándar: Monte Carlo (1000000 repetitions). "Cifras sujetas a cambios, por las continuas revisiones.

Fuente: elaborada con datos publicados por la Secretaría de Economía.

En el sureste, el estado que recibió mayor IED fue Quintana Roo, véase cuadro 13. Si bien, esta región no recibe un gran flujo de IED a los estados que el conforman, es importante notar que Campeche es el estado con menor atracción de IED durante 1999-2013. Particularmente, el referido estado requiere una política industrial que genere mayores eslabonamientos al interior de sus industrias para impulsar su economía además de que, con base en la muestra estimada, la variabilidad de la tasa de crecimiento del PIB real que explica la IED en Campeche es muy pequeña. Uno de los estados, de esta región, que explica un mayor porcentaje la variabilidad de la tasa de crecimiento del PIB real es Yucatán, alrededor de $1.2 \%$, véase cuadro 14 . 


\section{Cuadro 13}

sureste de México

\begin{tabular}{|lccc|}
\hline Entidad federativa & $\begin{array}{c}\text { IED, 1999-2013 } \\
\text { (millones de } \\
\text { dólares) }\end{array}$ & $\begin{array}{c}\text { Población } \\
(\mathbf{2 0 1 0}) \mathbf{I}^{\mathbf{1}}\end{array}$ & $\begin{array}{c}\text { Superficie } \\
\left(\mathbf{k m}^{\mathbf{2}} \mathbf{/ n}^{\mathbf{2}}\right.\end{array}$ \\
\hline Campeche & 41.2 & 822.441 & 57.727 \\
Quintana Roo/ ${ }^{3}$ & 3743.0 & 1325.578 & 42.535 \\
Tabasco & 817.2 & 2238.603 & 24.747 \\
Yucatán & 588.2 & 1955.577 & 39.671 \\
\hline
\end{tabular}

${ }^{*}$ Cifras sujetas a cambios, por las continuas revisiones. ${ }^{1 /}$ INEGI: Censo de Población y Vivienda 2010. 2/ INEGI. Marco geo-estadístico 2005.

$3 /$ No incluye la superficie de la isla Cozumel, la cual es de $498 \mathrm{~km}^{2}$. No incluye la superficie de la isla Mujeres, la cual es de $5 \mathrm{~km}^{2}$.

Fuente: elaborada con datos publicados por la Secretaría de Economía e INEGI.

\section{Cuadro 14}

Descomposición de la varianza del $\Delta$ PIBreal $^{*}$ sureste de México

\begin{tabular}{|cccccc|}
\hline Periodo & PIB_real & $\begin{array}{c}\text { IED_} \\
\text { Campeche }\end{array}$ & $\begin{array}{c}\text { IED_ } \\
\text { Quintana } \\
\text { Roo }\end{array}$ & $\begin{array}{c}\text { IED_- } \\
\text { Tabasco }\end{array}$ & $\begin{array}{c}\text { IED_ } \\
\text { Yucatan }\end{array}$ \\
\hline 1 & 100.0000 & 0.000000 & 0.000000 & 0.000000 & 0.000000 \\
2 & 99.31626 & 0.002588 & 0.030010 & 0.002829 & 0.648309 \\
3 & 98.26724 & 0.002641 & 0.131276 & 0.632188 & 0.966653 \\
4 & 98.02762 & 0.051785 & 0.298969 & 0.653168 & 0.968455 \\
5 & 97.42483 & 0.056799 & 0.636548 & 0.663271 & 1.218548 \\
6 & 96.94690 & 0.064593 & 0.824723 & 0.736667 & 1.427119 \\
7 & 96.85845 & 0.092878 & 0.827181 & 0.762445 & 1.459052 \\
8 & 96.80380 & 0.106827 & 0.863490 & 0.762718 & 1.463167 \\
\hline
\end{tabular}

Cholesky Ordering: PIB_real, IED_Campeche, IED_Quintana Roo, IED_Tabasco. Errores estándar: Monte Carlo (10 00000 repetitions). ${ }^{*}$ Cifras sujetas a cambios, por las continuas revisiones. Fuente: elaborada con datos publicados por la Secretaría de Economía. 
La región suroeste presenta montos bajos de IED; véase el cuadro 15. Sin embargo, Guerrero explica considerablemente la variabilidad de la tasa de crecimiento del PIB real, véase el cuadro 16. Lo que muestra un área de oportunidad para las autoridades, porque podrían impulsar en mayor medida las bondades de esta región.

Cuadro 15

Suroeste de México

\begin{tabular}{|lccc|}
\hline $\begin{array}{c}\text { Entidad } \\
\text { federativa }\end{array}$ & $\begin{array}{c}\text { IED, 1999-2013* } \\
\text { (Millones de } \\
\text { dólares) }\end{array}$ & Población (2010)/ ${ }^{\mathbf{1}}$ & Superficie $\mathbf{( k m}^{\mathbf{2}) /{ }^{\mathbf{2}}}$ \\
\hline Chiapas & 187.0 & 4796.580 & 73.681 \\
Guerrero & 468.3 & 3388.768 & 63.618 \\
Oaxaca & 289.1 & 3801.962 & 93.343 \\
\hline
\end{tabular}

"Cifras sujetas a cambios, por las continuas revisiones. ${ }^{1 /}$ INEGI: Censo de Población y Vivienda 2010. ² INEGI. Marco Geo-estadístico 2005.

Fuente: elaborada con datos publicados por la Secretaría de Economía e INEGI.

\author{
Cuadro 16 \\ Descomposición de la varianza $\triangle$ PIBreal $^{*}$ \\ Suroeste de México
}

\begin{tabular}{|ccccc|}
\hline Periodo & PIB_real & ED_Guerrero & IED_Oaxaca & IED_Chiapas \\
\hline 1 & 100.0000 & 0.000000 & 0.000000 & 0.000000 \\
2 & 81.70814 & 18.24351 & 0.048346 & 2.371258 \\
3 & 72.40186 & 27.55620 & 0.041943 & 2.345412 \\
4 & 71.02018 & 28.94156 & 0.038253 & 2.384386 \\
5 & 71.13982 & 28.82153 & 0.038652 & 2.384238 \\
6 & 71.17635 & 28.78435 & 0.039301 & 2.384992 \\
7 & 71.12643 & 28.83413 & 0.039441 & 2.384998 \\
8 & 71.09841 & 28.86217 & 0.039414 & 2.385013 \\
\hline
\end{tabular}

Cholesky Ordering: PIB_real, IED_Guerrero, IED_Oaxaca, IED_Chiapas. Errores estándar: Monte Carlo (1,000,000 repetitions). "Cifras sujetas a cambios, por las continuas revisiones. Fuente: elaborada con datos publicados por la Secretaría de Economía. 
En un esfuerzo por examinar con mayor profundidad la relación entre el crecimiento económico y la IED en México, así mismo se explorarán las Sociedades de IED por Entidad Federativa en la siguiente sección.

\section{Sociedades de IED por origen y destino}

El conteo de las sociedades que publica la Secretaría de Economía (SE) se actualiza constantemente. Véanse los cuadros de conteo de sociedades por origen en el apéndice $B$ y por estado de la república en el apéndice $C$.

En el apéndice B, de sociedades por origen, se observa que EU, España, Canadá, Países Bajos, Argentina, Alemania, Italia, República de Corea, Francia, Reino Unido, Colombia, China, Suiza, Venezuela, Japón, Brasil, Panamá, Chile, entre otros países, presentaron un mayor número de sociedades con flujos de IED hacia México en el periodo 1999-2013. ${ }^{2}$ Lo anterior, no necesariamente corresponde con el orden de los diez primeros países que enviaron un monto mayor de IED, en millones de dólares, el ranking es: EU (168 176.7), Países Bajos (46 124.5), España (42 915.6), Canadá (17 648.9), Bélgica (14 726.7), Reino Unido (8 984.1), Suiza (7 905.9), Alemania (7 575.5), Japón (7 556.5) y Luxemburgo (4 787.7). Esto indica que para los primeros países el número y el monto de IED corresponden en cierta medida, posteriormente no es así, por ejemplo Luxemburgo sólo tiene 377 SMIE (vigésimo en la lista de países por número de sociedades que enviaron IED a México), pero el monto de IED enviado es fuerte (por monto es el décimo país). De lo anterior se puede inferir que el número de eslabonamientos derivados de la IED por compañía podría hacerse de manera más eficiente.

En el apéndice $C$ se muestra el conteo de las Sociedades Mexicanas con Inversión Extranjera (SMIE) por Entidad Federativa de destino, durante 1999-2013. Una SMIE es considerada si se realizó un movimiento de IED en esa entidad y en caso de cambio de domicilio de la planta se contabiliza en el otro estado. Es decir, la sociedad se cuenta en el estado donde se

\footnotetext{
${ }^{2}$ Con respecto al país de origen es importante notar que la metodología del conteo considera el número de accionistas por países de origen. Particularmente, los datos indican que el número de sociedades que presentaron flujos de IED hacia México, por país de origen durante 1999-2013, provienen de EU (25 609), España (4 837), Canadá (3 355), Países Bajos (2 020), Argentina (1 723), Alemania (1 685), Italia (1 670), República de Corea (1 624), Francia (1 503) y Reino Unido (1 420).
} 
realiza la Inversión. El número de sociedades que presentaron flujos de IED hacia México, por Entidad Federativa durante 1999-2013, principalmente, se observó en: el Ciudad de México (22 574), Baja California (3 527), Estado de México (3 115), Baja California Sur (3 111) y Nuevo León (3 021).

Con base en la información anterior se puede concluir que el número de sociedades y montos coincide en los primeros países y Estados, después se disipa el efecto del número de sociedades que presentan flujos de IED. La relación entre el número y monto por país y estado podría tender a crear un mayor número de eslabonamientos.

\section{Conclusiones}

En la presente investigación se analizó el origen y los estados destino de la IED para el caso mexicano. Esto con el propósito de identificar posibles áreas de oportunidad para que las autoridades correspondientes implementen medidas de política económica que impulsen el crecimiento económico de México. Identificamos cuáles fueron los países que enviaron IED a México, durante 1999-2013, así como los estados de la república mexicana que recibieron el impulso de dicha inversión. Particularmente, analizamos la descomposición de varianza por regiones de la IED sobre la tasa de crecimiento del PIB real, durante 1999-2013.

Si bien México es una economía emergente atractiva que presenta una fuerte entrada de inversión de portafolio por sus condiciones macroeconómicas estables con este análisis se resalta que México también recibió montos considerables de IED, durante 1999-2013. Estos fueron, principalmente, provenientes de EU. Países Bajos, España, Canadá, Bélgica, Reino Unido, Suiza, entre otros países. Esto indica que México también es un destino de inversión de largo plazo, la referida inversión conlleva la introducción de nuevas tecnologías, el mejoramiento de plantas y la creación de eslabonamientos para las regiones económicas.

Para dimensionar qué región del país requiere de un mayor impulso para incrementar su actividad, realizamos un análisis de descomposición de varianza, el cual nos indica que, durante 1999-2013, la región que recibió mayor IED es la centro-sur que contiene a la Ciudad de México (primer receptor de IED) y el Estado de México (tercer receptor de IED). Sin embargo, la variabilidad de la tasa de crecimiento del PIB real explicada por 
los Estados que integran la región Centro-Sur es relativamente pequeña, en comparación con la variabilidad del crecimiento económico que explica la IED en Puebla. Esto posiblemente es resultado de los eslabonamientos entre sectores hacia donde se dirige la IED en la Ciudad de México. Los principales sectores, en orden de monto, que reciben IED en la Ciudad de México son: el sector servicios financieros y de seguros, el manufacturero, el comercio, servicios inmobiliarios y de alquiler de bienes muebles e intangibles, así como el sector de la información en medios masivos. Mientras que, los sectores en los que Puebla recibe mayor IED son: el sector manufacturero, servicios financieros y de seguros, comercio e información en medios masivos. Lo anterior muestra que el principal sector hacia donde se dirige la IED en Puebla es el sector manufacturero antes que el sector servicios y de seguros, este último es el primero para la Ciudad de México. Esto sugiere que el sector servicios y de seguros en la Ciudad de México requiere crear eslabonamientos intersectores para que la IED que tiene por destino a la Ciudad de México explique una mayor proporción de la variabilidad en la tasa de crecimiento del PIB real de México.

También se encontró que la región noreste de México recibe un monto considerable de IED. Dicha región contiene a Nuevo León (segundo receptor de IED). Los flujos de IED a Nuevo León se dirigen principalmente al sector manufacturero, servicios financieros y de seguros, comercio y al sector de servicios inmobiliarios y de alquiler de bienes muebles e intangibles. Esto muestra un rol activo del sector manufacturero. Dado este hecho, el diseño de política para la IED debería impulsar las regiones que no cuentan con atracción de IED y focalizar aquellas que contribuyen con la tasa de crecimiento del PIB real. De acuerdo con UNCTAD (2013) se deberían desarrollar políticas conjuntas entre inversión, comercio y servicios. Además se deben llevar a cabo enlaces regionales para que se generen spillovers productivos de las regiones con fuerte recepción de IED hacia las que aún no cuentan con ésta, esto impulsará el desarrollo Industrial de las regiones circundantes dado que el efecto de las interacciones entre el capital humano y las nuevas tecnologías incrementando con ello la productividad. 


\section{Referencias}

Basave, J y M. T. Gutiérrez (2011). "Mexico's Global Players. Great Diversity of Outward-Investing Industries in 2008 and the Impact of the Global Crisis on Mexican MNEs Varies by Industry in 2009". In: MNEs from Emerging Markets: New Players in the World FDI Market. Vale Columbia Center on Sustainable International Investment, Columbia University, New York, pp. 239-296.

Choe, J. I. (2003). "Do Foreign Direct Investment and Gross Domestic Investment Promote Economic Growth?" Review of Development Economics, 7(1), 44-57.

Ericsson, J. and I. Manuchehr (2001a). "On the Causality between Foreign Direct Investment and Output: a Comparative Study". International Trade Journal, 15(1), 1-26.

Márquez-Pozos J. M.; A. Islas-Camargo y F. Venegas-Martínez (2003). "Corrientes internacionales de capital e inversión extranjera de cartera: el caso de México 1989-1999". El Trimestre Económico, 70(4), 280, pp. 791-833.

Nair-Reichert, U., and D. Weinhold (2001). "Causality Tests for Cross-Country Panels: a New Look at FDI and Economic Growth in Developing Countries". Oxford Bulletin of Economics and Statistics, 63(2), pp. 153-171.

Nunnenkamp, P. and J. E. A. Bremont (2007). FDI in Mexico: An Empirical Assessment of Employment Effects (No. 1328). Kiel Institute for the World Economy (IfW).

Romero, J. (2012). "Inversión extranjera directa y crecimiento económico en México: 1940-2011". Investigación Económica, 71 282, pp. 109-147.

Shaikh, F. M. (2010). "Causality Relationship between Foreign Direct Investment, Trade and Economic Growth in Pakistan". In International Business Research, vol. 1, pp. 11-18. Harvard Business School.

Toda, H. Y., and Yamamoto, T. (1995). "Statistical Inference in Vector Autoregressions with Possibly Integrated Processes". Journal of Econometrics, 66(1), pp. 225-250.

UNCTAD, 2013. "World Investment Report 2013". New York and Geneva: The United Nations.

Wang, J. Y., and Blomström, M. (1992). "Foreign Investment and Technology Transfer: A simple Model". European Economic Review, 36(1), pp. 137-155.

Waldkirch, A. (2010). "The Effects of Foreign Direct Investment in Mexico since NAFTA". The World Economy, 33(5), pp. 710-745.

Yamada, H., and H. Y. Toda (1998). "Inference in Possibly Integrated Vector Autoregressive Models: Some Finite Sample Evidence". Journal of Econometrics, 86(1), pp. 55-95.

Yeaple, S. R. (2005). "A Simple Model of Firm Heterogeneity, International Trade, and Wages". Journal of International Economics, 65(1), pp. 1-20. 


\begin{tabular}{|c|c|c|c|c|c|c|c|c|c|c|c|c|c|c|c|c|c|c|c|c|c|c|c|c|c|}
\hline & 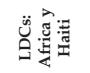 & 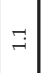 & 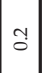 & oे & $\stackrel{\Upsilon}{-}$ & $\stackrel{\circ}{-}$ & $\stackrel{\leftrightarrow}{-}$ & 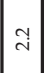 & $\stackrel{?}{-}$ & $\stackrel{\sim}{\rightarrow}$ & $\exists$ & o & $\begin{array}{l}\infty \\
0 \\
0\end{array}$ & $\Rightarrow$ & $\because$ & $\ddot{0}$ & $\mid \begin{array}{l}\infty \\
0 \\
0\end{array}$ & İ & $\stackrel{m}{\circ}$ & $\because 4$ & $\stackrel{0}{\circ}$ & フ̃. & $\stackrel{0}{0}$ & $\stackrel{2}{\circ}$ & $\stackrel{m}{0}$ \\
\hline & 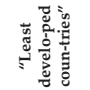 & $\stackrel{\sim}{\sim}$ & 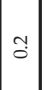 & $\dot{o}$ & $\stackrel{\leftrightarrow}{\rightarrow}$ & $\stackrel{\odot}{-}$ & $\exists$ & $\stackrel{m}{i}$ & $\stackrel{+}{-}$ & $\stackrel{\circ}{-}$ & $\stackrel{\sim}{\Im}$ & $\stackrel{\odot}{-}$ & $\stackrel{\infty}{0}$ & $\stackrel{\sim}{\rightarrow}$ & $\because$ & $\ddot{0}$ & $\begin{array}{l}\infty \\
0 \\
0\end{array}$ & ?3. & $\stackrel{3}{0}$ & $\ddot{0}$ & $\hat{o}$ & 3 & $\stackrel{\odot}{-}$ & oे & $\stackrel{\infty}{\infty}$ \\
\hline & 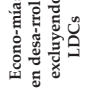 & $\hat{\grave{\lambda}}$ & 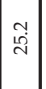 & $\begin{array}{l}\text { ते } \\
\text { ते }\end{array}$ & $\begin{array}{l}\infty \\
\stackrel{\vec{j}}{ }\end{array}$ & 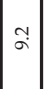 & 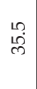 & 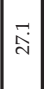 & $\mid \begin{array}{l}+ \\
\stackrel{d}{\sim}\end{array}$ & $\begin{array}{l}0 \\
\stackrel{i}{i}\end{array}$ & $\begin{array}{l}\stackrel{\rho}{\infty} \\
\stackrel{\infty}{-}\end{array}$ & $\begin{array}{l}\infty \\
\stackrel{\sim}{\sim}\end{array}$ & $\begin{array}{l}\grave{\infty} \\
\ddot{\infty}\end{array}$ & \begin{tabular}{|l|}
$\mathcal{Y}$ \\
$\mathcal{Z}$
\end{tabular} & 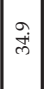 & $\begin{array}{l}n \\
\stackrel{n}{\dot{m}}\end{array}$ & $\begin{array}{l}0 \\
\dot{\sim} \\
\end{array}$ & $\begin{array}{l}\dot{0} \\
\stackrel{\infty}{\sim}\end{array}$ & \begin{tabular}{|l|}
$\stackrel{0}{\circ}$ \\
$\stackrel{\leftrightarrow}{\circ}$
\end{tabular} & \begin{tabular}{|c|} 
\\
$\infty$ \\
$-\infty$ \\
\end{tabular} & 롱 & 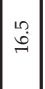 & $\stackrel{\leftrightarrow}{i}$ & $\stackrel{\sim}{M}$ & 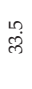 \\
\hline 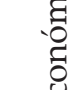 & 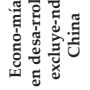 & $\begin{array}{l}\stackrel{a}{ } \\
\dot{\sim}\end{array} \mid$ & 芦 & $\overrightarrow{\mathrm{i}}$ & 离 & 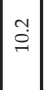 & \begin{tabular}{|l|}
0 \\
$\stackrel{0}{0}$ \\
0
\end{tabular} & $\begin{array}{l}\ddot{\Delta} \\
\ddot{i}\end{array}$ & 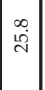 & ปֶ. & $\overrightarrow{\dot{\sim}}$ & $\stackrel{\leftrightarrow}{\sim}$ & $\overrightarrow{\text { fं }}$ & $\stackrel{f}{f}$ & $\vec{\infty}$ & $\underset{\sim}{\stackrel{D}{\sim}}$ & $\frac{\partial}{\vec{N}}$ & ڤุ. & $\begin{array}{l}\stackrel{2}{+} \\
\dot{I}\end{array}$ & 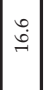 & $\stackrel{\circ}{\stackrel{+}{ \pm}}$ & 혹 & ते & 获 & $\overrightarrow{\mathrm{N}}$ \\
\hline 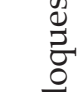 & 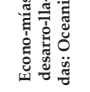 & $\stackrel{\infty}{\wedge}$ & $\begin{array}{c}a \\
\infty\end{array}$ & $\stackrel{\circ}{\curvearrowright}$ & 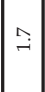 & $\mid$\begin{tabular}{|c|}
$n$ \\
0
\end{tabular} & $\tilde{N}$ & $\begin{array}{l}0 \\
\end{array}$ & $\mid \begin{array}{l}\infty \\
+ \\
+\end{array}$ & ڤ̂े & $\stackrel{+}{+}$ & $F$ & $\stackrel{\circ}{\circ}$ & $\begin{array}{l}7 \\
+\end{array}$ & $\begin{array}{ll}7 \\
6\end{array}$ & $\stackrel{\leftrightarrow}{-}$ & $\stackrel{\mathcal{I}}{\mp}$ & $\hat{\sigma}$ & $\stackrel{\circ}{+}$ & in & $\stackrel{m}{+}$ & Fे & $\begin{array}{l}\infty \\
i \\
i\end{array}$ & ن. & ले \\
\hline$\frac{O}{\varrho}$ & 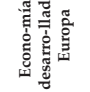 & 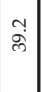 & $\begin{array}{l}\infty \\
\stackrel{\vec{f}}{+}\end{array}$ & $\exists$ & $\overrightarrow{7}$ & 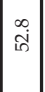 & $\mid \begin{array}{l}\infty \\
\stackrel{\infty}{\infty} \\
\end{array}$ & $\begin{array}{l}\stackrel{a}{\circ} \\
\stackrel{m}{m}\end{array}$ & \begin{tabular}{|c|}
0 \\
$\dot{\leftrightarrow}$
\end{tabular} & ֻุ. & $\begin{array}{ll}\infty \\
\dot{F} \\
\dot{F}\end{array}$ & 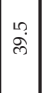 & $\stackrel{m}{\stackrel{i}{i}}$ & 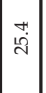 & $\begin{array}{l}\hat{\rho} \\
\dot{\rho}\end{array}$ & $\stackrel{\circ}{ \pm}$ & $\begin{array}{l}0 \\
\dot{m} \\
\end{array}$ & ڤั. & \begin{tabular}{|l|}
$n$ \\
ஸे
\end{tabular} & $\mid \begin{array}{l}\hat{\sigma} \\
\dot{\phi}\end{array}$ & \begin{tabular}{|l|}
$\mathscr{2}$ \\
$\mathscr{\mathcal { H }}$
\end{tabular} & \begin{tabular}{|l|}
$\ddot{1}$ \\
in
\end{tabular} & 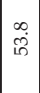 & 吕 & $\begin{array}{l}\infty \\
\text { m. } \\
\text { m. }\end{array}$ \\
\hline$\stackrel{\widetilde{\sigma}}{ \pm}$ & 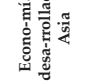 & 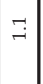 & $\stackrel{9}{-}$ & $\stackrel{9}{-}$ & $\stackrel{2}{\circ}$ & $\cong$ & $\stackrel{\circ}{-}$ & $\hat{o}$ & $\because$ & $ت$ & $\begin{array}{l}0 \\
0\end{array}$ & $\mid$ & $\ddot{0}$ & $\mid \begin{array}{l}\infty \\
0 \\
0\end{array}$ & \begin{tabular}{|c|} 
\\
-
\end{tabular} & 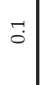 & $\stackrel{\rightarrow}{\rightarrow}$ & $\ddot{0}$ & $\stackrel{\circ}{-}$ & $\begin{array}{r}-1 \\
\dot{1}\end{array}$ & \begin{tabular}{|c|} 
\\
$i$ \\
$i$ \\
$i$
\end{tabular} & ò & $\stackrel{\circ}{\circ}$ & $\stackrel{\infty}{\rightarrow}$ & $\stackrel{0}{0}$ \\
\hline 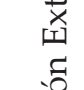 & 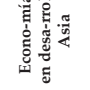 & Ḧ & $\begin{array}{l}\circ \\
i\end{array}$ & 10 & $\begin{array}{l}0 \\
\infty\end{array}$ & \begin{tabular}{|l|}
$\hat{\sigma}$ \\
$\dot{\varphi}$ \\
\end{tabular} & $\begin{array}{l}\infty \\
\stackrel{\infty}{\Omega}\end{array}$ & $\stackrel{m}{\wedge}$ & 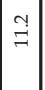 & $\stackrel{+}{ت}$ & in & $\stackrel{\odot}{-}$ & $\vec{\sigma}$ & \begin{tabular}{|l|}
$n$ \\
ते
\end{tabular} & \begin{tabular}{|l|} 
\\
$\dot{\sim}$
\end{tabular} & $\stackrel{\overbrace{}}{\sim}$ & $\hat{\sigma}$ & $\stackrel{\text { so }}{\circ}$ & $\mid \begin{array}{c}\infty \\
\propto \\
\sigma\end{array}$ & $\stackrel{\circ}{=}$ & $\begin{array}{l}\infty \\
\infty \\
\infty\end{array}$ & $\stackrel{\ominus}{0}$ & 官 & $\stackrel{\partial}{\partial}$ & च्ञें \\
\hline 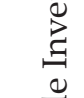 & 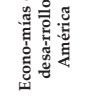 & $\stackrel{\stackrel{\mathrm{I}}{\mathrm{I}}}{ }$ & $\begin{array}{l}0 \\
\stackrel{\circ}{\sim} \\
.\end{array}$ & ف & $\begin{array}{l}\hat{\mathrm{j}} \\
\end{array}$ & 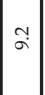 & $\mid \begin{array}{c}\stackrel{\sim}{\sim} \\
\stackrel{\sim}{\sim}\end{array}$ & $\underset{⿱}{\stackrel{m}{+}}$ & $\stackrel{\substack{n \\
=}}{=}$ & 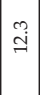 & $\stackrel{H}{=}$ & $\stackrel{9}{\vec{z}}$ & $\stackrel{+}{\mathrm{I}}$ & $\overrightarrow{\mathrm{I}}$ & 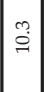 & $\stackrel{\circ}{\wedge}$ & $ت$ & 苑 & $\underset{\sim}{\mathscr{7}}$ & \begin{tabular}{|l|}
0 \\
$i$
\end{tabular} & $\underset{+}{+}$ & $\underset{⿱ 乛}{+}$ & $\stackrel{\stackrel{\leftrightarrow}{\circ}}{\wedge}$ & $\hat{a}$ & $\stackrel{\infty}{0}_{0}^{0}$ \\
\hline 䒕 & 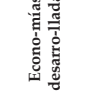 & $\vec{\Sigma}$ & $\mid \begin{array}{l}0 \\
\stackrel{2}{R}\end{array}$ & 至 & $\mid \begin{array}{l}\stackrel{9}{\mathrm{i}} \\
\end{array}$ & $\mid \begin{array}{l}\infty \\
\infty \\
\infty\end{array}$ & $\mid \begin{array}{l}10 \\
8 \\
3\end{array}$ & $\mid \stackrel{\circ}{\circledR}$ & $\begin{array}{l}\mathcal{Z} \\
\mathbb{2}\end{array}$ & 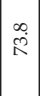 & $\frac{\dot{2}}{2}$ & $\begin{array}{l}\overrightarrow{0} \\
\infty\end{array}$ & \begin{tabular}{|l}
10 \\
100 \\
10
\end{tabular} & $\begin{array}{l}0 \\
\stackrel{2}{1}\end{array}$ & 동 & $\overrightarrow{8}$ & 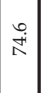 & $\begin{array}{l}\infty \\
\infty \\
\infty\end{array}$ & $\mid \begin{array}{l}0 \\
\dot{+} \\
\infty\end{array}$ & $\underset{\dot{\infty}}{+}$ & $\begin{array}{l}m \\
\stackrel{\infty}{\infty} \\
\infty\end{array}$ & \begin{tabular}{|c|}
$\infty$ \\
$\infty$ \\
$\infty$
\end{tabular} & $\vec{Z}$ & के & Iี \\
\hline $\begin{array}{l}\frac{\pi}{0} \\
0 \\
0\end{array}$ & 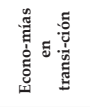 & $\stackrel{8}{0}$ & 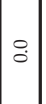 & 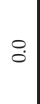 & $\stackrel{0}{\circ}$ & $\stackrel{0}{\circ}$ & $\because$ & $\stackrel{1}{\circ}$ & $\stackrel{0}{\circ}$ & $\because$ & $:$ & $:$ & $\because$ & $\because$ & $\stackrel{0}{\circ}$ & $\because$ & $:$ & $\because$ & $\because$ & $\because$ & $\because$ & $\because$ & $\overrightarrow{0}$ & $\stackrel{\circ}{\circ}$ & $\stackrel{+}{\rightarrow}$ \\
\hline 苹 & 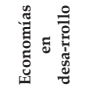 & $\stackrel{\leftrightarrow}{\dot{\infty}}$ & 苦 & $\overrightarrow{\dot{a}}$ & 록 & 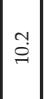 & 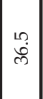 & $\mid$\begin{tabular}{l|}
$\ddot{2}$ \\
$\ddot{2}$
\end{tabular} & 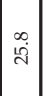 & તૃ. & ت्ञ & $\begin{array}{l}\infty \\
\stackrel{\infty}{\sim} \\
\stackrel{0}{\mid}\end{array}$ & \begin{tabular}{|l|}
$n$ \\
लें
\end{tabular} & \begin{tabular}{|c|}
\multirow{2}{*}{} \\
字
\end{tabular} & के & 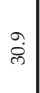 & 苟 & $\stackrel{m}{\stackrel{\infty}{\infty}}$ & $\begin{array}{l}\stackrel{\rho}{2} \\
\stackrel{2}{2}\end{array}$ & 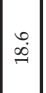 & $\begin{array}{l}\hat{2} \\
\stackrel{2}{2}\end{array}$ & \begin{tabular}{|c|}
0 \\
$\dot{0}$ \\
-1
\end{tabular} & เ્ & స్త & 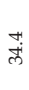 \\
\hline 질 & $\frac{}{\frac{\pi}{2}}$ & 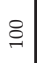 & $\stackrel{-}{-}$ & $\stackrel{-}{\circ}$ & $\stackrel{\square}{\circ}$ & $\stackrel{-}{\circ}$ & 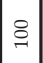 & $\stackrel{\circ}{\circ}$ & $\stackrel{-}{\circ}$ & $\stackrel{-}{\circ}$ & $\stackrel{.}{\circ}$ & 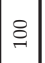 & $\stackrel{-}{\circ}$ & $\stackrel{-}{\circ}$ & $\stackrel{-}{-}$ & 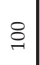 & 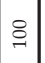 & $\stackrel{-}{\circ}$ & 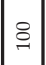 & $\stackrel{-}{\circ}$ & $\stackrel{8}{-}$ & $\stackrel{8}{-}$ & $\stackrel{\odot}{-}$ & $\stackrel{ }{\circ}$ & $\underset{-1}{8}$ \\
\hline 㤩 & & : & 点 & S̃ & \begin{tabular}{|l}
0 \\
2 \\
$\sigma$
\end{tabular} & \begin{tabular}{|l|}
\multirow{2}{2}{} \\
2
\end{tabular} & $\mid \begin{array}{c}12 \\
2 \\
2\end{array}$ & \begin{tabular}{|l}
2 \\
2 \\
2
\end{tabular} & 点 & $\begin{array}{l}\infty \\
\infty \\
0 \\
0\end{array}$ & $\begin{array}{l}2 \\
\stackrel{2}{\sigma}\end{array}$ & 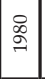 & \begin{tabular}{|l|}
$\vec{\Phi}$ \\
$\stackrel{\sigma}{\Xi}$
\end{tabular} & 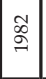 & \begin{tabular}{|c|}
$\mathscr{2}$ \\
$\stackrel{2}{\sigma}$
\end{tabular} & 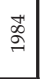 & $\begin{array}{l}\infty \\
\stackrel{2}{\circ}\end{array}$ & $\stackrel{\circ}{\circ}$ & $\begin{array}{l}\stackrel{\circ}{\infty} \\
\stackrel{2}{\circ}\end{array}$ & 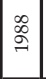 & $\begin{array}{l}\stackrel{2}{\circ} \\
\stackrel{2}{2}\end{array}$ & ঃ & $\bar{\sigma}$ & $\stackrel{\text { }}{\sigma}$ & $\sigma^{\infty}$ \\
\hline
\end{tabular}




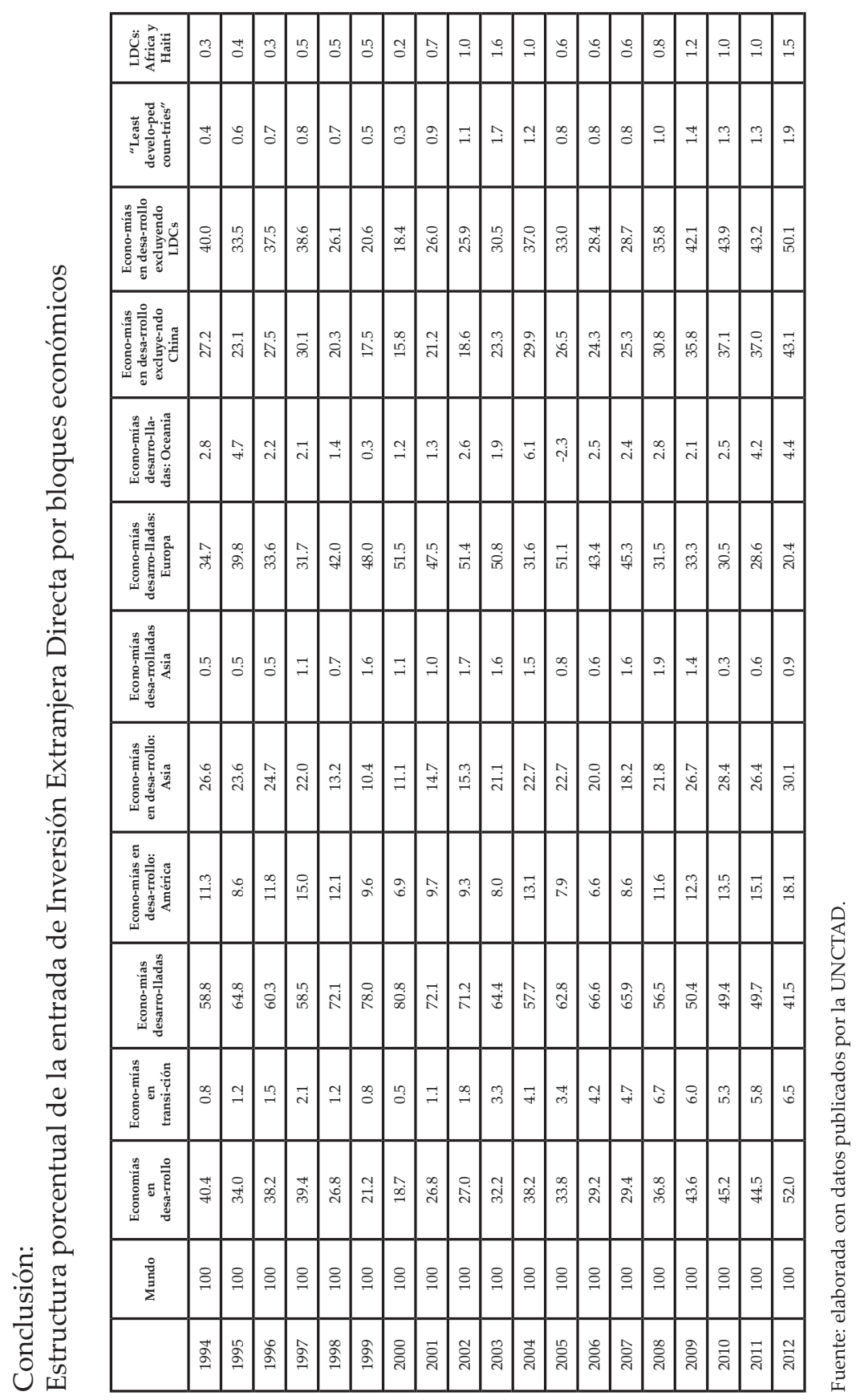




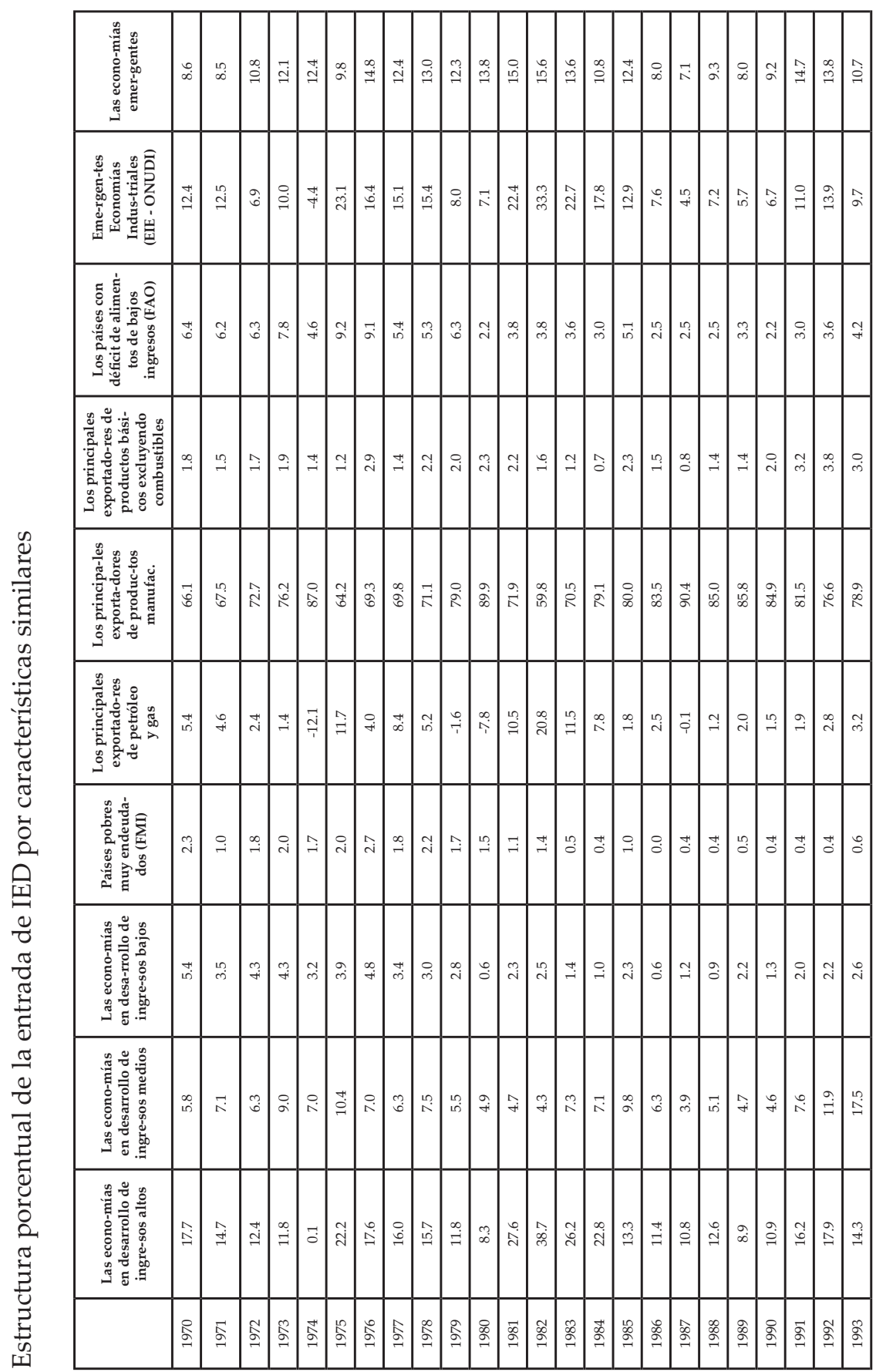




\begin{tabular}{|c|c|c|c|c|c|c|c|c|c|c|c|c|c|c|c|c|c|c|c|}
\hline 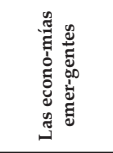 & 苞 & $\stackrel{\circ}{\underset{+}{+}}$ & $\begin{array}{l}\stackrel{+}{\text { 曷 }} \\
\end{array}$ & 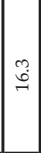 & $\stackrel{\vartheta}{\ominus}$ & $\stackrel{\infty}{\stackrel{0}{\varrho}}$ & 華 & $\begin{array}{l}\infty \\
\stackrel{\infty}{\oplus}\end{array}$ & $\stackrel{\overbrace{}}{\stackrel{\rho}{\varrho}}$ & $\begin{array}{l}\infty \\
\stackrel{\infty}{=}\end{array}$ & ले & 苟 & $\underset{\infty}{+}$ & $\infty$ & $\stackrel{\infty}{\infty}$ & $\underset{\infty}{\infty}$ & $\begin{array}{l}\stackrel{2}{2} \\
\stackrel{2}{2}\end{array}$ & $\stackrel{\grave{\mathrm{j}}}{ }$ & $\stackrel{\sharp !}{\circ}$ \\
\hline 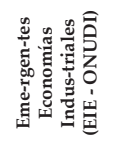 & $\vec{m}$ & తે & $\begin{array}{l}\stackrel{\leftrightarrow}{\leftrightarrow} \\
\stackrel{\leftrightarrow}{n}\end{array}$ & $\vec{\infty}$ & $\begin{array}{l}\stackrel{n}{\mathrm{i}} \\
\mathrm{J}\end{array}$ & $\begin{array}{l}0 \\
\sigma \\
\sigma\end{array}$ & gे & $\overrightarrow{\mathrm{I}}$ & $\stackrel{0}{\text { I }}$ & $\overrightarrow{\mathrm{I}}$ & $\begin{array}{l}\stackrel{10}{1} \\
\stackrel{2}{n}\end{array}$ & $\begin{array}{l}a \\
\stackrel{a}{2}\end{array}$ & 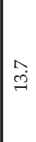 & 엄 & $\begin{array}{l}0 \\
\stackrel{0}{\sigma}\end{array}$ & $\vec{\infty}$ & 吕 & 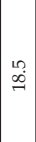 & ㅇํㄹ \\
\hline 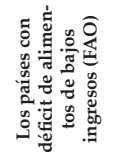 & $\begin{array}{c}\infty \\
\infty \\
\infty\end{array}$ & $\stackrel{\odot}{\infty}$ & $F$ & 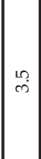 & $\stackrel{\sim}{\rightarrow}$ & \begin{tabular}{|l}
$\infty$ \\
0
\end{tabular} & 吕 & $\stackrel{\text { בִ }}{\rightarrow}$ & i̊ & $\begin{array}{l}0 \\
i\end{array}$ & $\vec{\infty}$ & $\underset{F}{F}$ & $\stackrel{\circ}{\rightarrow}$ & $\begin{array}{l}\infty \\
\infty \\
\text { ले }\end{array}$ & in & $\hat{o}$ & ${ }_{10}^{\circ}$ & $\begin{array}{l}+1 \\
0\end{array}$ & $\stackrel{\infty}{\wedge}$ \\
\hline 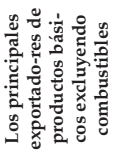 & F & F & $\stackrel{\leftrightarrow}{+}$ & \begin{tabular}{|c|c|}
$\infty$ \\
$\infty$
\end{tabular} & ปี & $\stackrel{m}{m}$ & $\cong$ & $\stackrel{\infty}{\circ}$ & $\stackrel{?}{-}$ & بْ & $\overrightarrow{\mathrm{i}}$ & 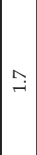 & $\stackrel{\text { Ing }}{\longrightarrow}$ & $\stackrel{+}{-}$ & $\stackrel{\text { i }}{\text { in }}$ & $\stackrel{\infty}{-}$ & $\vec{i}$ & $\hat{\mathrm{i}}$ & $\stackrel{m}{+}$ \\
\hline 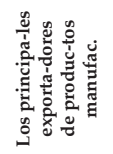 & 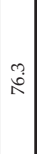 & $\stackrel{n}{\stackrel{n}{*}}$ & $\stackrel{2}{+}$ & $\stackrel{\circ}{\dot{K}}$ & 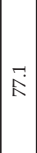 & $\begin{array}{l}0 \\
\infty \\
\infty\end{array}$ & $\begin{array}{l}0 \\
\dot{\infty} \\
\dot{\infty}\end{array}$ & $\frac{n}{\infty}$ & 望 & $\begin{array}{l}\circ \\
\text { ほ }\end{array}$ & 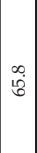 & $\begin{array}{l}\stackrel{\rho}{\hat{~}} \\
\end{array}$ & \begin{tabular}{|l}
0 \\
0 \\
6
\end{tabular} & 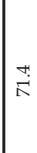 & $\stackrel{\circ}{\mathrm{b}}$ & $\begin{array}{l}\hat{\circ} \\
i n \\
i n\end{array}$ & $\overrightarrow{\mathrm{d}}$ & î̀. & $\stackrel{+}{\vec{H}}$ \\
\hline 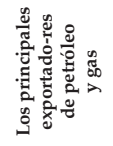 & $\stackrel{c}{\sim}$ & $\hat{\mathrm{i}}$ & $\stackrel{m}{\circ}$ & $\stackrel{\circ}{+}$ & $\stackrel{3}{\mathrm{i}}$ & $\stackrel{-1}{-}$ & $\stackrel{m}{-}$ & הี & $\begin{array}{l}\infty \\
\text { i }\end{array}$ & กี & 70 & $\begin{array}{l}0 \\
0 \\
0\end{array}$ & $\begin{array}{l}\infty \\
0 \\
0\end{array}$ & જ. & ळे & $\stackrel{?}{=}$ & $\because$ & $\stackrel{\curvearrowright}{\curvearrowright}$ & $\stackrel{\circ}{\infty}$ \\
\hline 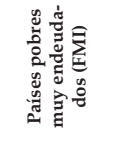 & 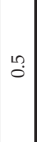 & 每 & $\stackrel{10}{\circ}$ & $\hat{o}$ & $\hat{o}$ & $\stackrel{\text { In }}{\circ}$ & 3 & $\stackrel{0}{0}$ & $\stackrel{\circ}{\rightarrow}$ & $\stackrel{\sim}{\rightarrow}$ & $\stackrel{\circ}{-}$ & $\stackrel{\circ}{-}$ & \begin{tabular}{|l}
$\infty$ \\
0
\end{tabular} & $\stackrel{\infty}{0}$ & $\Xi$ & $\stackrel{\llcorner}{\rightarrow}$ & 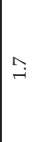 & $\stackrel{-}{-}$ & $\stackrel{\circ}{\mathrm{i}}$ \\
\hline 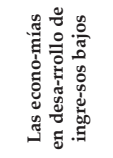 & बे & $\stackrel{H}{i}$ & $\vec{i}$ & $\hat{\mathrm{i}}$ & $\mid \begin{array}{r}0 \\
-1\end{array}$ & $\dot{0}$ & $\hat{o}$ & بْ & $\stackrel{\stackrel{n}{\mathrm{~N}}}{\mathrm{~N}}$ & $\begin{array}{l}\infty \\
i \\
ن\end{array}$ & $\stackrel{\mathscr{D}}{\mathrm{i}}$ & $\hat{\mathrm{i}}$ & $\begin{array}{l}0 \\
\text { ले }\end{array}$ & $\vec{m}$ & $\overrightarrow{\text { in }}$ & $\overrightarrow{6}$ & $\stackrel{+}{+}$ & $\overrightarrow{\text { in }}$ & : \\
\hline 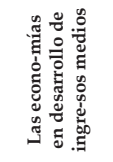 & $\begin{array}{l}\infty \\
\infty \\
-\end{array}$ & $\begin{array}{l}\text { 吕 } \\
\stackrel{10}{n}\end{array}$ & $\stackrel{0}{\stackrel{0}{\circ}}$ & $\underset{+}{\stackrel{+}{ \pm}}$ & $\begin{array}{l}\infty \\
\sigma \\
\sigma\end{array}$ & \begin{tabular}{|l}
0 \\
10 \\
10
\end{tabular} & $\begin{array}{l}\infty \\
\infty \\
\dot{2}\end{array}$ & $\begin{array}{l}0 \\
\infty\end{array}$ & ঙ & $\widehat{\stackrel{\mathrm{J}}{ }}$ & בี & \begin{tabular}{|l}
$\stackrel{n}{\mathrm{i}}$ \\
$\mathrm{i}$
\end{tabular} & 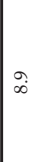 & $\stackrel{\searrow}{\curvearrowright}$ & $\stackrel{\sim}{0}$ & $\begin{array}{l}0 \\
\stackrel{\mathrm{i}}{ }\end{array}$ & さे & $\overrightarrow{\mathrm{I}}$ & 苞 \\
\hline \multirow[t]{2}{*}{ 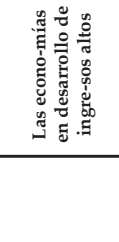 } & $\begin{array}{l}\infty \\
\infty \\
\stackrel{\infty}{\sim}\end{array}$ & ָુ & 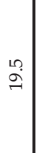 & ते & $\begin{array}{l}m \\
\stackrel{2}{n} \\
\stackrel{2}{n}\end{array}$ & 于 & I & $\stackrel{m}{\stackrel{?}{\sim}}$ & 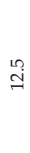 & $\hat{\imath}$ & \begin{tabular}{|l}
10 \\
$\tilde{i}$ \\
$\tilde{i}$
\end{tabular} & \begin{tabular}{|c|}
0 \\
$\stackrel{\infty}{\sigma}$ \\
\end{tabular} & $\stackrel{?}{\stackrel{2}{2}}$ & 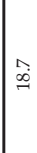 & $\stackrel{\text { n? }}{\mathrm{N}}$ & $\mid \begin{array}{l}\infty \\
\stackrel{+}{+}\end{array}$ & $\begin{array}{l}0 \\
\stackrel{i}{d}\end{array}$ & $\begin{array}{l}\tilde{3} \\
\stackrel{3}{2}\end{array}$ & 今े \\
\hline & 菏 & 尽 & $\begin{array}{l}\stackrel{2}{\sigma} \\
\stackrel{2}{2}\end{array}$ & 参 & $\stackrel{\infty}{\circ}$ & 高 & ఫి & ઠేి & ઠิ & ¿ి & ठ્ત & 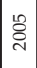 & ఫ్రి & స్సి & 㝕 & ठ્તે & 올 & $\overline{\vec{c}}$ & సี \\
\hline
\end{tabular}




\begin{tabular}{|c|c|c|c|c|c|c|c|c|c|c|c|c|c|c|c|c|c|c|c|}
\hline 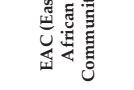 & 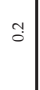 & "5 & 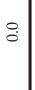 & 5 & $\overrightarrow{0}$ & $\overrightarrow{0}$ & 3 & 15 & 3 & $\begin{array}{l}3 \\
0\end{array}$ & $\overrightarrow{0}$ & 5 & $\overrightarrow{0}$ & $\therefore$ & 5 & $\because$ & $:$ & $\stackrel{8}{0}$ & $:$ \\
\hline 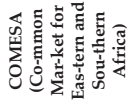 & $\begin{array}{r}\circ \\
\dot{r}\end{array}$ & $\stackrel{\circ}{-}$ & 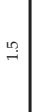 & :̊) & $\stackrel{\odot}{-}$ & ? & $\stackrel{d}{m}$ & : & 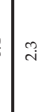 & 菅 & $\stackrel{8}{0}$ & $\because$ & 5 & $\stackrel{\rightleftarrows}{-}$ & $\overrightarrow{\mathrm{i}}$ & $\infty_{0}^{\infty}$ & o & gे & $\stackrel{\circ}{\circ}$ \\
\hline 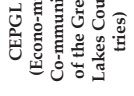 & $:$ & 5 & 5 & -1 & $\begin{array}{c}3 \\
8\end{array}$ & $\therefore 7$ & $\exists$ & 8 & I & \begin{tabular}{|c|}
0 \\
0
\end{tabular} & 3 & 8 & 菅 & $\therefore$ & $\begin{array}{l}z \\
:\end{array}$ & 8 & 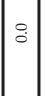 & $\therefore$ & $: 8$ \\
\hline 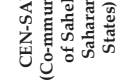 & is & ले & $\infty$ & $\vec{i}$ & $\cong$ & $\stackrel{9}{\circ}$ & के & $\overrightarrow{\mathrm{j}}$ & ए & 穴 & i & $\pi$ & 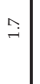 & $\vec{i}$ & 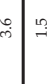 & $\exists$ & $\stackrel{9}{-1}$ & $\tilde{\lambda}$ & $\stackrel{7}{=}=$ \\
\hline 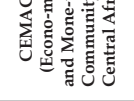 & $\because$ & $\stackrel{103}{8}$ & $\stackrel{1}{\circ}$ & $\stackrel{20}{8}$ & $\hat{\circ}$ & $\dot{\partial}$ & 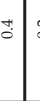 & $\vdots$ & 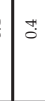 & $\begin{array}{l}3 \\
0\end{array}$ & 3 & $\dddot{2}$ & $\stackrel{\infty}{\infty}$ & 5 & 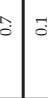 & O & 3 & $\bar{\phi}$ & $:$ \\
\hline 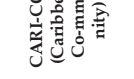 & $\stackrel{\circ}{\mathrm{i}}$ & $\vec{i}$ & i & $\stackrel{?}{\rightarrow}$ & $\stackrel{9}{-}$ & 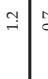 & $\hat{\circ}$ & $\dot{0}$ & I & $\stackrel{2}{\circ}$ & ?ֶ. & : & ت艹 & รี & $: 8$ & 7 & $:$ & 5 & $\therefore$ \\
\hline 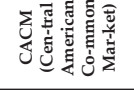 & $\stackrel{\circ}{\circ}$ & $\stackrel{20}{0}$ & 范 & 8 & 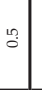 & ôे & $\stackrel{8}{8}$ & 8 & I & 3 & 3 & $\tilde{O}$ & $\stackrel{m}{0}$ & z & 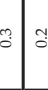 & I & 3 & $\overline{0}$ & $\overrightarrow{0}$ \\
\hline 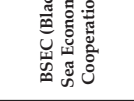 & $\stackrel{\infty}{\circ}$ & $\stackrel{\circ}{\circ}$ & 잉 & $\hat{o}$ & $\stackrel{10}{\circ}$ & $\stackrel{8}{\circ}$ & 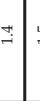 & 2 & : & $\stackrel{?}{-1}$ & 8 & $\stackrel{\infty}{\circ}$ & $\stackrel{\circ}{\rightarrow}$ & $\Xi$ & $\stackrel{a}{a}$ & $\stackrel{\circ}{\circ}$ & $\stackrel{\infty}{0}$ & $\hat{o}$ & : \\
\hline 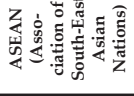 & में & $\overrightarrow{~ ल े ~}$ & 品 & 8 & iे & $\vec{\infty}$ & $\approx$. & $\stackrel{f}{i} \dot{f}$ & : & $\dot{+}$ & ำ & בु & : & $\overrightarrow{\text { in }}$ & $\vec{F}$ & ल & $\stackrel{+}{+}$ & ले & ુુ \\
\hline 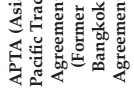 & $\stackrel{\infty}{\circ}$ & $\stackrel{\circ}{\circ}$ & 芦 & $\because$ & $\stackrel{m}{3}$ & 3 & 3 & 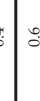 & 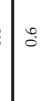 & : & $\hat{o}$ & $\exists$ & ì & $\infty$ & $\vec{F}$ & 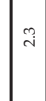 & $\mid \begin{array}{l}0 \\
\mathrm{i}\end{array}$ & $\mathbb{i}^{\sharp}$ & $\tilde{N}$ \\
\hline 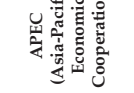 & बे & 今े & 㘶 & 离 & ङु & $\begin{array}{c}n \\
m \\
m\end{array}$ & 过 & 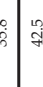 & 范 & \begin{tabular}{|l|}
$\hat{0}$ \\
$\hat{n}$
\end{tabular} & 管 & $\mid \begin{array}{l}\infty \\
\dddot{q}\end{array}$ & ڤี่ & 茴 & \begin{tabular}{l|l} 
\\
$b^{\circ}$
\end{tabular} & $\begin{array}{l}2 \\
6 \\
6\end{array}$ & $\begin{array}{l}3 \\
i \\
i n\end{array}$ & స్తి & $\begin{array}{l}\infty \\
\neq \\
\neq\end{array}$ \\
\hline 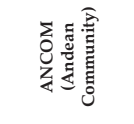 & $\stackrel{9}{-}$ & $\stackrel{+}{I}$ & 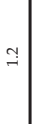 & $:$ & $\dot{\circ}$ & $\stackrel{\circ}{-1}$ & 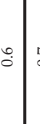 & 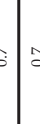 & 10 & $\stackrel{\circ}{\circ}$ & $\stackrel{\infty}{\circ}$ & $\begin{array}{l}\infty \\
0 \\
\infty\end{array}$ & $\stackrel{+}{-}$ & $\stackrel{\odot}{-}$ & त) & म & $\%$ & ت艹 & ت艹 \\
\hline 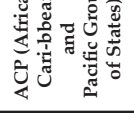 & $\stackrel{+}{\stackrel{0}{\circ}}$ & $\infty$ & $\therefore$ & 3 & $\stackrel{2}{\therefore}$ & $\overrightarrow{0}$ & $\begin{array}{c}\infty \\
i\end{array}$ & $y$ & 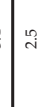 & $\stackrel{+}{-}$ & $\stackrel{p}{\infty}$ & ले & $\stackrel{\text { i̊ }}{\text { s. }}$ & $\tilde{i}$ & त : & 9 & $\cong$ & $\stackrel{\infty}{\rightarrow}$ & $\cong$ \\
\hline & 尺̊ & 尽 & న్ & $\stackrel{0}{\circ}$ & 芯 & 点 & 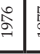 & 2 & $\underbrace{2}_{2}$ & \begin{tabular}{|l|}
$\circ$ \\
$\stackrel{\circ}{\circ}$
\end{tabular} & $\begin{array}{l}\overrightarrow{0} \\
\stackrel{0}{\sim}\end{array}$ & \begin{tabular}{|l}
$\infty$ \\
$\stackrel{\infty}{\sim}$
\end{tabular} & $\stackrel{\mathscr{B}}{\triangle}$ & 范 & $\begin{array}{ll}0 \\
2 \\
2 \\
2\end{array}$ & $\mid \begin{array}{l}0 \\
\text { 总 }\end{array}$ & 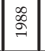 & 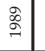 & ఏ \\
\hline
\end{tabular}




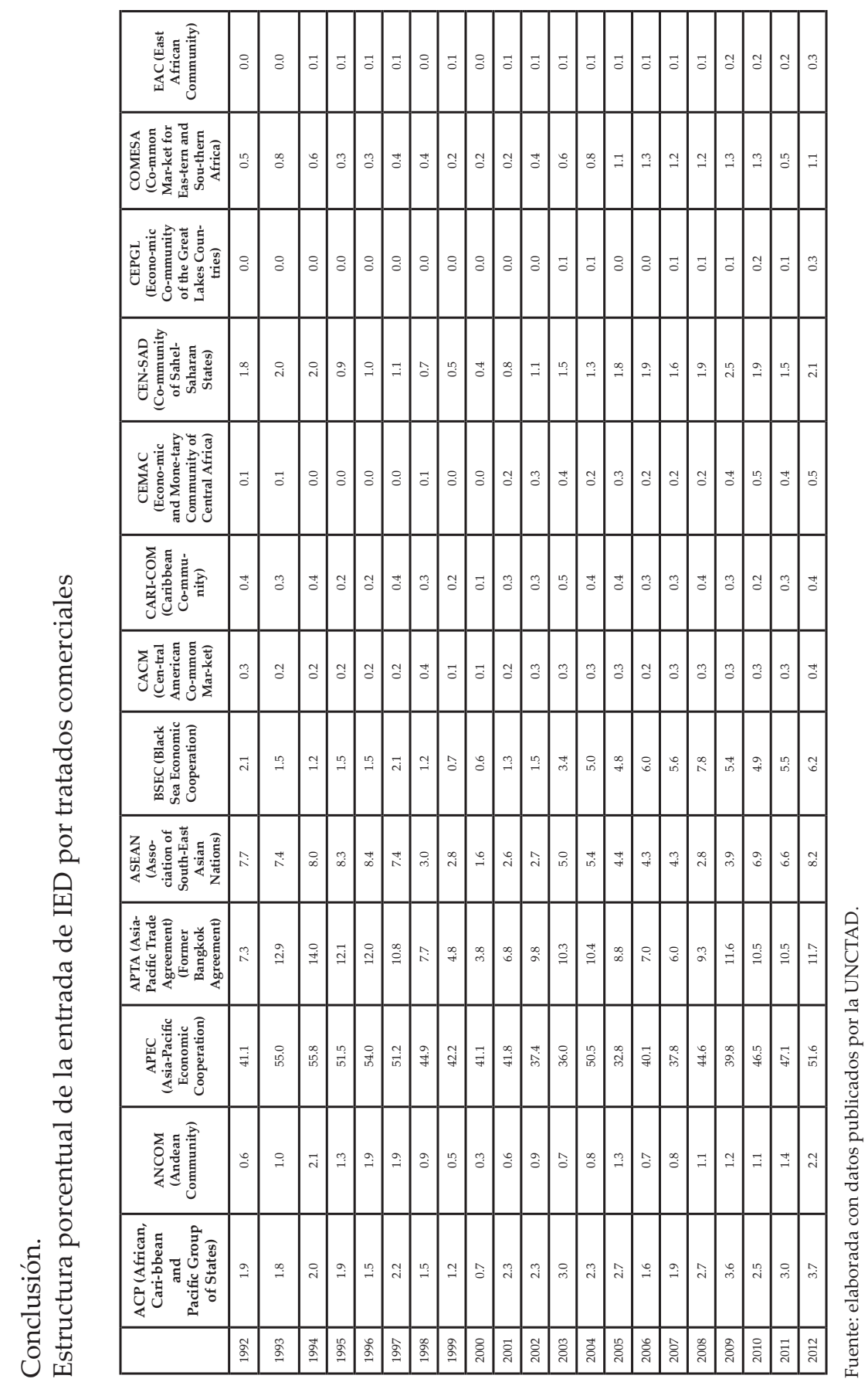




\begin{tabular}{|c|c|c|c|c|c|c|c|c|c|c|c|c|c|c|c|c|c|c|c|c|c|}
\hline 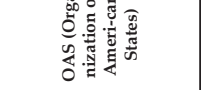 & \begin{tabular}{|l|}
$\overrightarrow{0}$ \\
00
\end{tabular} & $\begin{array}{l}\infty \\
\dot{8} \\
8\end{array}$ & $\mid \begin{array}{l}* \\
\text { à }\end{array}$ & $\mid \begin{array}{c}0 \\
⿱ 亠 䒑 \\
\infty\end{array}$ & $\begin{array}{l}0 \\
\dot{\infty} \\
\dot{c}\end{array}$ & $\overrightarrow{80}$ & 宗 & 常 & बे & $\begin{array}{ll}7 \\
7\end{array}$ & 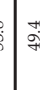 & $\mid \begin{array}{l}3 \\
\stackrel{0}{\infty}\end{array}$ & $\frac{m}{2}$ & 吕 & 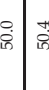 & $\begin{array}{l}\infty \\
\dot{B}\end{array}$ & 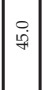 & 荘 & $\stackrel{7}{m}$ & 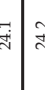 & \\
\hline 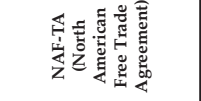 & 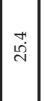 & $\underset{\text { I }}{\mathcal{A}}$ & $\stackrel{+}{i}$ & iे & $\stackrel{m}{\vec{m}}$ & $\vec{d}$ & वे & 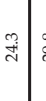 & $\begin{array}{l}\infty \\
\dot{\infty} \\
\dot{\infty}\end{array}$ & \begin{tabular}{c}
4 \\
\hdashline \\
\hdashline
\end{tabular} & $\stackrel{\leftrightarrow}{F}$ & 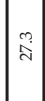 & $\stackrel{m}{\bar{m}}$ & $\vec{b}$ & สิ & $\begin{array}{l}n \\
15\end{array}$ & $\underset{7}{7}$ & $\stackrel{\circ}{\circ}$ & $\begin{array}{l}3 \\
\dot{S}\end{array}$ & $\underset{\sim}{\infty}$ & \\
\hline 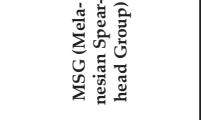 & 1 & $\stackrel{+}{0}$ & $\stackrel{\circ}{\circ}$ & $\stackrel{0}{\circ}$ & : & $\begin{array}{ll}5 & 3\end{array}$ & 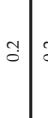 & 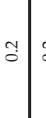 & รี & 5 & $\approx$ & oี & : & 3 & \begin{tabular}{c|c}
0 \\
\end{tabular} & 5 & $\because$ & 5 & 5 & 50 & \\
\hline 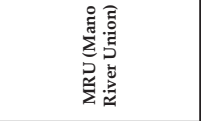 & 今. & $\overrightarrow{\dot{Q}}$ & $\dot{0}$ & $\stackrel{0}{\circ}$ & 苛 & $\rightarrow$ & $\stackrel{0}{\circ}$ & $\hat{\circ}$ & \begin{tabular}{c|c}
$\hat{\delta}$ & 2 \\
\end{tabular} & $\begin{array}{l}3 \\
3\end{array}$ & 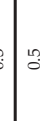 & $\stackrel{0}{\circ}$ & 今̃ & $\overrightarrow{0}$ & 8 & $=0$ & ב̃ & 苟 & 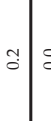 & $8:$ & \\
\hline 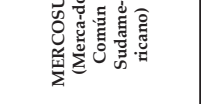 & \begin{tabular}{|l|} 
\\
\end{tabular} & $\stackrel{l}{i}$ & $\because$ & iे & $\stackrel{\circ}{\infty}$ & $\begin{array}{lll}\hat{b} & 3\end{array}$ & $\infty$ & $\begin{array}{l}\infty \\
\infty\end{array}$ & $\stackrel{\infty}{\wedge}$ & $\begin{array}{rlll}-10 \\
\end{array}$ & is & 3 & $\ddot{\oplus}$ & $\stackrel{\infty}{\longrightarrow}$ & +2 & $\rightarrow$ & $\overrightarrow{\mathrm{i}}$ & $\underset{-}{ \pm}$ & $\stackrel{\infty}{\rightarrow}$ & i & \\
\hline 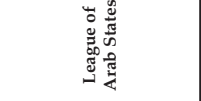 & 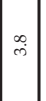 & $\stackrel{\infty}{0}$ & $\stackrel{\infty}{\rightarrow}$ & $\stackrel{m}{\dot{p}}$ & $\vec{F}$ & $\stackrel{\circ}{\circ}$ & $\stackrel{\infty}{\rightarrow}$ & $\stackrel{m}{m}$ & $\stackrel{2}{\rightarrow}$ & $\begin{array}{l}\overrightarrow{2} \\
\vec{b}\end{array}$ & $\stackrel{m}{\stackrel{\rho}{\rho}}$ & ते & $\stackrel{\stackrel{m}{=}}{=}$ & $\Xi$ & 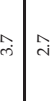 & 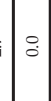 & $\stackrel{\oplus}{-}$ & $\hat{o}$ & $\stackrel{-10}{-}$ & 2 & \\
\hline 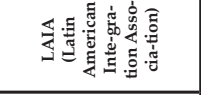 & $\mid \begin{array}{l}\infty \\
\infty\end{array}$ & $\stackrel{+}{\stackrel{0}{口}}$ & $\stackrel{m}{\oplus}$ & 2 & $\hat{0}$ & $\stackrel{s}{=}$ & $\widehat{\mathrm{I}}$ & $\stackrel{\infty}{\circ}$ & $\stackrel{\text { }}{\stackrel{c}{0}}$ & $\stackrel{\infty}{\circ}$ & $\stackrel{ \pm}{=}$ & $\stackrel{\Xi}{\Rightarrow}$ & 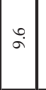 & is & 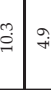 & {$\left[\begin{array}{l}\infty \\
\infty\end{array}\right.$} & in & $F$ & $\stackrel{\infty}{\infty}$ & 6. & \\
\hline 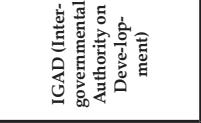 & \begin{tabular}{|c|} 
\\
\end{tabular} & $\overrightarrow{0}$ & $\overrightarrow{0}$ & $\ddot{8}$ & 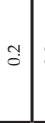 & $\stackrel{1}{5}$ & $\stackrel{3}{0}$ & $\stackrel{3}{0}$ & $\overrightarrow{0}$ & 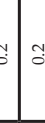 & 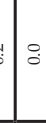 & 5 & 8 & $\stackrel{8}{\circ}$ & $: 8$ & 5 & $\because$ & 8 & $\therefore$ & $: 2$ & \\
\hline 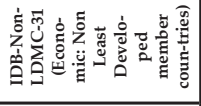 & $\begin{array}{l}n \\
\infty\end{array}$ & iे & 足 & 㸃 & $\stackrel{0}{i}$ & $\stackrel{2}{\beth}$ & $\stackrel{+!}{\infty}$ & $\stackrel{\infty}{\infty}$ & $\begin{array}{l}10 \\
\infty\end{array}$ & $\begin{array}{c}y \\
2\end{array}$ & $\stackrel{\vec{g}}{9}$ & $\begin{array}{l}m \\
\dot{Z} \\
\end{array}$ & $\mid \begin{array}{l}0 \\
\dot{\oplus}\end{array}$ & 苜 & 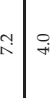 & 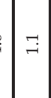 & $\ddot{i}$ & $\vec{m}$ & 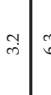 & 62 & \\
\hline 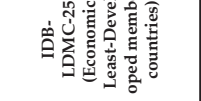 & 范 & ֻั & $\tilde{O}$ & 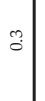 & $\stackrel{0}{\circ}$ & $\therefore$ & 范 & 范 & $\stackrel{0}{\circ}$ & \begin{tabular}{l|ll} 
\\
$B$
\end{tabular} & 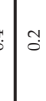 & 3 & 8 & 5 & $\therefore$ & 5 & $\because$ & 5 & 52 & 8 & \\
\hline 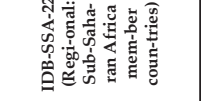 & $\overrightarrow{\mathrm{i}}$ & $\stackrel{\sim}{\mathrm{N}}$ & $\stackrel{\mathscr{n}}{\mathrm{N}}$ & $\vec{i}$ & $\stackrel{\bullet}{-}$ & i & ì & సี & 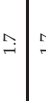 & $=$ & I & $\underset{-}{\sharp}$ & 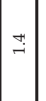 & $\stackrel{20}{\circ}$ & In: & $\infty_{\infty}^{\infty}$ & $\ddot{0}$ & $\stackrel{\leftrightarrow}{-}$ & $\stackrel{\circ}{\circ}$ & 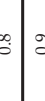 & \\
\hline 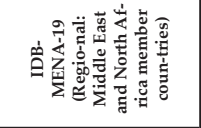 & 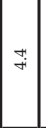 & $\exists$ & $\stackrel{\circ}{i}$ & $\begin{array}{l}m \\
i \\
i\end{array}$ & 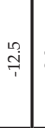 & $\stackrel{m}{\infty}$ & 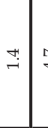 & F & 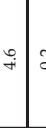 & 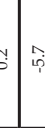 & $\stackrel{\stackrel{2}{g}}{=}$ & व̃ & $\cong$ & $\stackrel{m}{=}$ & $\left.\infty_{\infty}^{\infty}\right)$ & 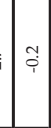 & $\stackrel{\leftrightarrow}{\rightarrow}$ & $\stackrel{\rightarrow}{\rightarrow}$ & 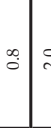 & 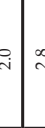 & \\
\hline 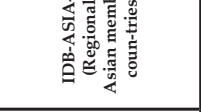 & $\stackrel{\sim}{i}$ & $\stackrel{\text { i }}{ }$ & $\stackrel{i}{i}$ & $\dot{m}$ & ले & $3:$ & is & $\begin{array}{l}* \\
\mathrm{~N}\end{array}$ & $\underset{\sim}{\infty}$ & $=\pi$ & $\dot{A}$ & $\vec{i}$ & $\stackrel{m}{m}$ & $\stackrel{\infty}{\rightarrow}$ & 2 & 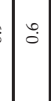 & \begin{tabular}{|c|}
$\infty$ \\
0 \\
0
\end{tabular} & $\exists$ & $\Rightarrow \Rightarrow$ & $\infty$ & \\
\hline & \begin{tabular}{|l|}
2 \\
\multirow{2}{2}{} \\
\end{tabular} & 疍 & \begin{tabular}{|l|} 
\\
S \\
\end{tabular} & 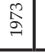 & \begin{tabular}{l|l} 
岕 \\
.
\end{tabular} & \begin{tabular}{l|l}
5 \\
2 \\
\end{tabular} & 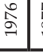 & $\begin{array}{c}\text { S } \\
\end{array}$ & 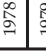 & \begin{tabular}{l|l}
8 \\
\hdashline \\
\hdashline
\end{tabular} & \begin{tabular}{l|l}
$\vec{\circ}$ \\
$\stackrel{\circ}{\circ}$
\end{tabular} & \begin{tabular}{|c|c|}
$\stackrel{0}{2}$ \\
$\stackrel{2}{2}$
\end{tabular} & 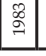 & 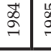 & \begin{tabular}{c}
80 \\
\hdashline \\
$\circ$
\end{tabular} & $\begin{array}{l}\infty \\
\stackrel{8}{\circ} \\
\end{array}$ & \begin{tabular}{|l|}
$\infty$ \\
$\stackrel{0}{\circ}$ \\
\end{tabular} & 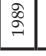 & $\begin{array}{l}2 \\
\end{array}$ & S. & \\
\hline
\end{tabular}




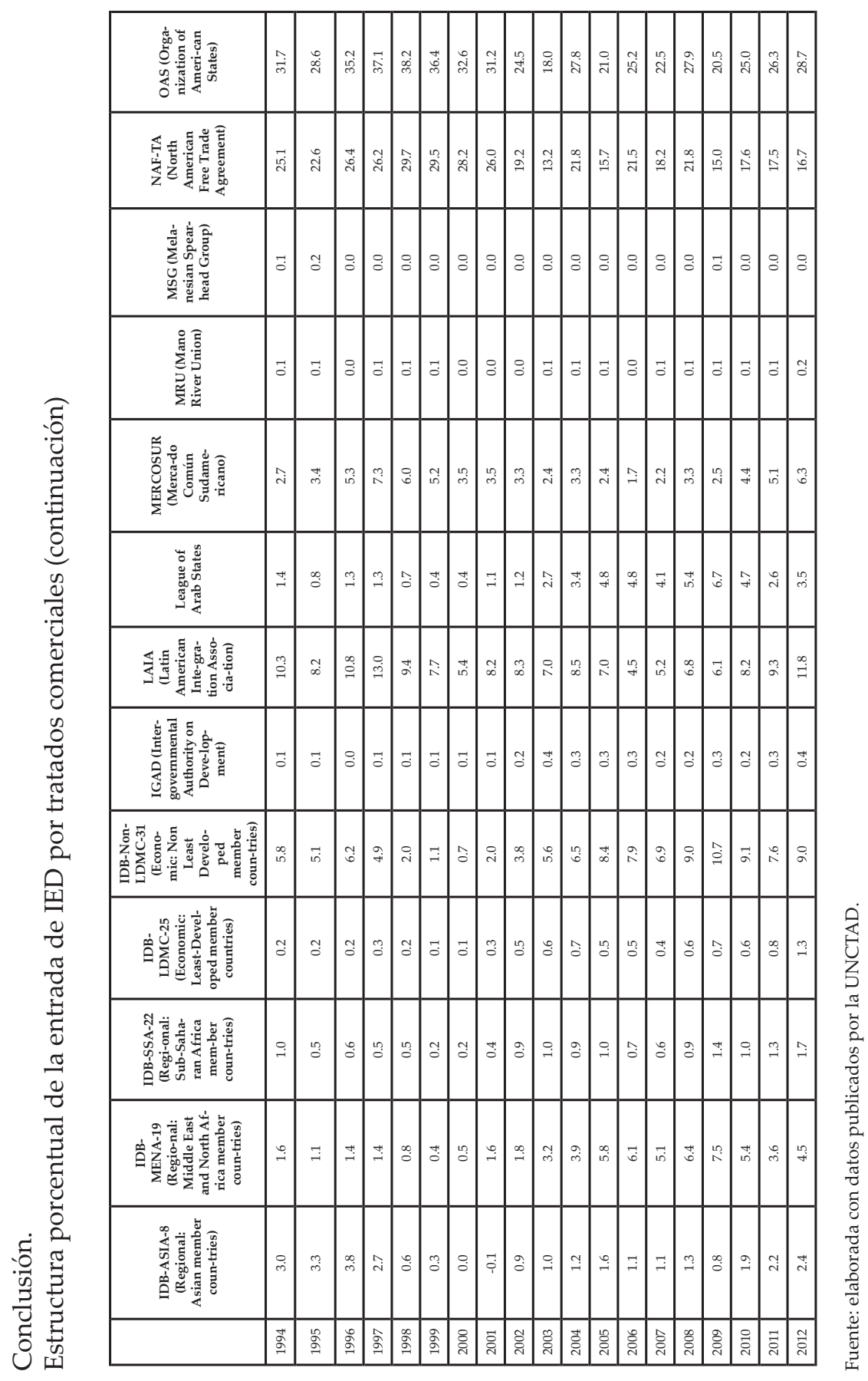




\section{Apéndice B}

Número de sociedades que presentaron flujos de IED hacia México por país de origen, $(1999-2013)^{*}$

\begin{tabular}{|c|c|}
\hline Estados Unidos de América & 25609 \\
\hline España & 4837 \\
\hline Canadá & 3355 \\
\hline Países Bajos & 2020 \\
\hline Argentina & 1723 \\
\hline Alemania & 1685 \\
\hline Italia & 1670 \\
\hline Corea & 1624 \\
\hline Francia & 1503 \\
\hline Reino Unido de la Gran Bretaña e Irlanda del Norte & 1420 \\
\hline Colombia & 1055 \\
\hline China, República Popular de & 846 \\
\hline Suiza & 804 \\
\hline Venezuela, República bolivariana de & 796 \\
\hline Japón & 783 \\
\hline Brasil & 617 \\
\hline Panamá & 568 \\
\hline Chile & 555 \\
\hline Luxemburgo & 377 \\
\hline Bélgica & 359 \\
\hline Uruguay & 329 \\
\hline Cuba & 311 \\
\hline Israel & 307 \\
\hline Perú & 299 \\
\hline Taiwán provincia de China & 269 \\
\hline Suecia & 253 \\
\hline Guatemala & 228 \\
\hline Dinamarca & 208 \\
\hline
\end{tabular}

\begin{tabular}{|c|c|}
\hline Costa Rica & 202 \\
\hline Singapur & 184 \\
\hline Australia & 181 \\
\hline Islas Caimán & 180 \\
\hline Austria & 168 \\
\hline India & 158 \\
\hline $\begin{array}{l}\text { Islas vírgenes de los Esta- } \\
\text { dos Unidos }\end{array}$ & 143 \\
\hline Irlanda & 134 \\
\hline Ecuador & 125 \\
\hline Islas Vírgenes Británicas & 116 \\
\hline Portugal & 116 \\
\hline Puerto Rico & 100 \\
\hline Bermudas & 89 \\
\hline El Salvador & 85 \\
\hline Belice & 76 \\
\hline Noruega & 76 \\
\hline Nueva Zelandia & 71 \\
\hline Bahamas, Las & 68 \\
\hline República Dominicana & 68 \\
\hline Paraguay & 65 \\
\hline Federación de Rusia & 64 \\
\hline $\begin{array}{l}\text { Corea, República Popular } \\
\text { Democrática de }\end{array}$ & 63 \\
\hline Liechtenstein & 60 \\
\hline $\begin{array}{l}\text { Hong Kong (RAE de } \\
\text { China) }\end{array}$ & 55 \\
\hline Turquía & 51 \\
\hline Honduras & 49 \\
\hline
\end{tabular}




\section{Continuación.}

Número de sociedades que presentaron flujos de IED hacia México por país de origen, (1999-2013)*

\begin{tabular}{|c|c|}
\hline Polonia & 47 \\
\hline Bolivia (Estado Plurinacional de) & 45 \\
\hline Finlandia & 43 \\
\hline Nicaragua & 42 \\
\hline República Checa & 37 \\
\hline Hungría & 33 \\
\hline Sudáfrica & 33 \\
\hline Barbados & 31 \\
\hline Antillas Holandesas & 30 \\
\hline Malasia & 29 \\
\hline Pakistán & 27 \\
\hline Líbano & 26 \\
\hline Grecia & 23 \\
\hline Irán, República Islámica de & 23 \\
\hline Egipto & 22 \\
\hline Filipinas & 21 \\
\hline Rumania & 20 \\
\hline Chipre & 18 \\
\hline Bulgaria & 17 \\
\hline Nigeria & 16 \\
\hline Serbia y Montenegro & 16 \\
\hline Ucrania & 14 \\
\hline Eslovaquia & 13 \\
\hline Siria, República Árabe de & 13 \\
\hline Armenia & 12 \\
\hline Tailandia & 12 \\
\hline Eslovenia & 11 \\
\hline Marruecos & 11 \\
\hline
\end{tabular}

\begin{tabular}{|c|c|}
\hline Congo, República Democrática del & 9 \\
\hline Islandia & 9 \\
\hline Malta & 9 \\
\hline Croacia & 8 \\
\hline Gibraltar & 8 \\
\hline Mauricio & 8 \\
\hline Dominica & 7 \\
\hline Mónaco & 7 \\
\hline Emiratos Árabes Unidos & 6 \\
\hline Haití & 6 \\
\hline Jamaica & 6 \\
\hline Sri Lanka & 6 \\
\hline Trinidad y Tobago & 6 \\
\hline Túnez & 6 \\
\hline Aruba & 5 \\
\hline Georgia & 5 \\
\hline Islas Turcas y Caicos & 5 \\
\hline Jordania & 5 \\
\hline Liberia & 5 \\
\hline Libia & 5 \\
\hline San Cristóbal y Nieves & 5 \\
\hline Vietnam & 5 \\
\hline Zambia & 5 \\
\hline Lituania & 4 \\
\hline Argelia & 3 \\
\hline Indonesia & 3 \\
\hline Kenia & 3 \\
\hline Seychelles & 3 \\
\hline
\end{tabular}




\section{Conclusión.}

Número de sociedades que presentaron flujos de IED hacia México por país de origen, (1999-2013)*.

\begin{tabular}{|l|c|}
\hline Albania & 2 \\
\hline Andorra & 2 \\
\hline Arabia Saudita & 2 \\
\hline Bangladesh & 2 \\
\hline Camerún & 2 \\
\hline Cisjordania y la Franja de Gaza (Palestina) & 2 \\
\hline Curazao & 2 \\
\hline Estonia & 2 \\
\hline Etiopía & 2 \\
\hline Irak & 2 \\
\hline Islas Marshall & 2 \\
\hline Kuwait & 2 \\
\hline Mauritania & 2 \\
\hline Qatar & 2 \\
\hline República Centroafricana & 2 \\
\hline Samoa & 1 \\
\hline Senegal & 1 \\
\hline Togo & 2 \\
\hline Zimbabue & 2 \\
\hline Angola & 1 \\
\hline Azerbaiyán & 1 \\
\hline Bahréin & 1 \\
\hline Belarús & 1 \\
\hline Camboya & 1 \\
\hline Comoras & 1 \\
\hline Fiyi, República de & 1 \\
\hline Ghana & 1 \\
\hline Granada & 1 \\
\hline Jersey & 1 \\
\hline Kazajstán, República de & 1 \\
\hline Letonia & 1 \\
\hline Maldivas & 1 \\
\hline Malí & 1 \\
\hline Micronesia, Estados Federados de & 2 \\
\hline Mongolia & 2 \\
\hline Surinam & 2 \\
\hline Yemen, República de & 2 \\
\hline
\end{tabular}

* Con información reportada al 31 de diciembre de 2013. Cifras sujetas a cambios, por las continuas revisiones. Fuente: elaborada con datos publicados por la Secretaría de Economía. 
Apéndice C

Número de sociedades que presentaron flujos de IED hacia México por entidad federativa (1999-2013)*

\begin{tabular}{|c|c|}
\hline \multicolumn{2}{|c|}{$\begin{array}{l}\text { Número de sociedades que presentaron flujos de IED hacia } \\
\text { México por entidad federativa } 1999-2013^{*}\end{array}$} \\
\hline Ciudad de México & 22574 \\
\hline Baja California & 3527 \\
\hline Estado de México & 3115 \\
\hline Baja California Sur & 3111 \\
\hline Nuevo León & 3021 \\
\hline Quintana Roo & 2957 \\
\hline Jalisco & 2829 \\
\hline Yucatán & 1560 \\
\hline Chihuahua & 1314 \\
\hline Querétaro & 1073 \\
\hline Guanajuato & 1034 \\
\hline Sonora & 993 \\
\hline Puebla & 856 \\
\hline Tamaulipas & 776 \\
\hline Coahuila & 634 \\
\hline Nayarit & 452 \\
\hline Veracruz & 446 \\
\hline San Luis Potosí & 370 \\
\hline Sinaloa & 357 \\
\hline Oaxaca & 343 \\
\hline Aguascalientes & 325 \\
\hline Morelos & 271 \\
\hline Durango & 232 \\
\hline Guerrero & 229 \\
\hline Michoacán & 159 \\
\hline Campeche & 150 \\
\hline Tabasco & 113 \\
\hline Colima & 107 \\
\hline Tlaxcala & 96 \\
\hline Zacatecas & 90 \\
\hline Chiapas & 85 \\
\hline Hidalgo & 82 \\
\hline
\end{tabular}

*Con información reportada al 31 de diciembre de 2013. Cifras sujetas a cambios, por las continuas revisiones.

Fuente: elaborada con datos publicados por la Secretaría de Economía. 
Conteo de las SMIEs por año

\begin{tabular}{|cc|}
\hline \multicolumn{2}{|c|}{$\begin{array}{c}\text { Número de sociedades que presentaron flujos } \\
\text { de IED hacia México por año }\end{array}$} \\
\hline Año & Número de sociedades \\
1999 & 674 \\
2000 & 7469 \\
2001 & 7240 \\
2002 & 7090 \\
2003 & 6982 \\
2004 & 7381 \\
2005 & 7658 \\
2006 & 7695 \\
2007 & 7640 \\
2008 & 6713 \\
2009 & 5569 \\
2010 & 5595 \\
2011 & 5820 \\
2012 & 5146 \\
2013 & 5045 \\
\hline
\end{tabular}

* Con información reportada al 31 de diciembre de 2013. Cifras sujetas a cambios, por las continuas revisiones.

Fuente: elaborada con datos publicados por la Secretaría de Economía. 